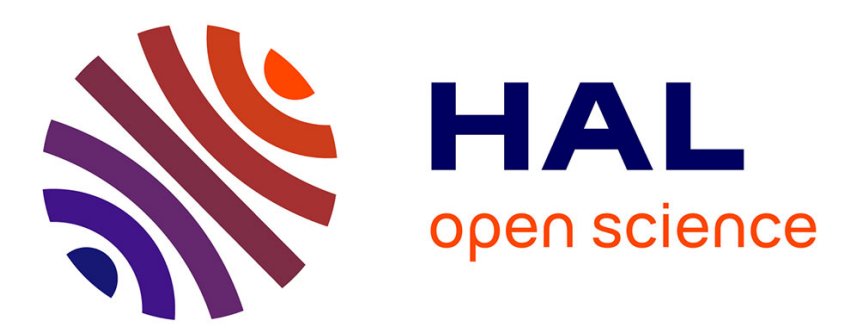

\title{
Sparse polynomial surrogates for non-intrusive, high-dimensional uncertainty quantification of aeroelastic computations
}

Eric Savin, Jean-Luc Hantrais-Gervois

\section{- To cite this version:}

Eric Savin, Jean-Luc Hantrais-Gervois. Sparse polynomial surrogates for non-intrusive, highdimensional uncertainty quantification of aeroelastic computations. Probabilistic Engineering Mechanics, 2020, 59, pp.103027. 10.1016/j.probengmech.2020.103027 . hal-02865271

\section{HAL Id: hal-02865271 \\ https://hal.science/hal-02865271}

Submitted on 11 Jun 2020

HAL is a multi-disciplinary open access archive for the deposit and dissemination of scientific research documents, whether they are published or not. The documents may come from teaching and research institutions in France or abroad, or from public or private research centers.
L'archive ouverte pluridisciplinaire HAL, est destinée au dépôt et à la diffusion de documents scientifiques de niveau recherche, publiés ou non, émanant des établissements d'enseignement et de recherche français ou étrangers, des laboratoires publics ou privés. 


\title{
Sparse polynomial surrogates for non-intrusive, high-dimensional uncertainty quantification of aeroelastic computations
}

\author{
Éric Savin ${ }^{\mathrm{a}, *}$, Jean-Luc Hantrais-Gervois ${ }^{\mathrm{b}}$ \\ ${ }^{a}$ ONERA/DTIS, Université Paris-Saclay, F-91123 Palaiseau, France \\ ${ }^{b}$ ONERA/DAAA, Université Paris-Saclay, F-92190 Meudon, France
}

\begin{abstract}
This paper is concerned with aircraft aeroelastic interactions and the propagation of parametric uncertainties in numerical simulations using high-fidelity fluid flow solvers. More specifically, the influence of variable operational and structural parameters (random inputs) on the drag performance and deformation (outputs) of a flexible wing in transonic regime, is assessed. Because of the complexity of fluid flow solvers, non-intrusive uncertainty quantification techniques are favored. Polynomial surrogate models based on homogeneous chaos expansions in the random inputs are commonly considered in this respect. The polynomial expansion coefficients are constructed using either structured sampling sets of the input parameters, as Gauss quadrature nodes, or unstructured sampling sets, as in Monte-Carlo methods. In complex systems such as the advanced aeroelastic test case studied here, the output quantities of interest generally depend only weakly on the multiple
\end{abstract}

\footnotetext{
*Corresponding author. Tel.: +330 146734 645; fax: +33 0146734143

Email addresses: eric.savin@onera.fr (Éric Savin), jean-luc.hantrais-gervois@onera.fr (Jean-Luc Hantrais-Gervois)
} 
cross-interactions between the random inputs. Consequently, only low-order polynomials significantly contribute to their surrogates, which thus have a sparse structure in the underlying polynomial bases. This feature prompts to use compressed sensing, or compressive sampling theory for the construction of the polynomial surrogates. The proposed methodology is non-adapted and considers unstructured sampling sets orders of magnitude smaller than the ones required by the usual techniques with structured sampling sets. It is illustrated in the present work for a moderately to high dimensional parametric space.

Keywords: Computational fluid dynamics, Aeroelasticity, Polynomial surrogates, Polynomial chaos, Compressed sensing.

\section{Introduction}

Aeroelasticity deals with the coupling of a flexible structure and a fluid flow and involves complex, possibly highly non-linear physical phenomena. The assessment of these non-linear effects is of crucial importance for the stability and strength of the structure, such as aircraft, bridge, turbine, etc., since they may lead to its complete destruction in case of self-sustained (limite-cycle) or divergent (flutter) oscillations. On an aircraft wing, fluid flows induce structural deformations which in turn influence the fluid dynamics such as for example shock behavior at transonic speeds, which in turn impacts the performance of the wing. Aeroelastic interactions have thus to be accounted for in design, testing, certification, and subsequent optimization

if need be. However several sources of variability arise in the prediction of aeroelastic phenomena, including manufacturing process, assembly, measure- 
ment uncertainties during ground or flight testing, parametric uncertainties, or model and numerical uncertainties pertaining to analysis methods. Uncertainty quantification (UQ) and the various techniques it encompasses are aimed at estimating quantitatively all those sources of uncertainties or variabilities and predicting their significance on quantities of interest used to design, test, certify or optimize the structure. UQ is generally addressed in a probabilistic framework, although the actual regulations do not rely on probabilities for certification but rather introduce the notion of risks related to undesirable (failure) scenarios. Recent reviews on the various issues raised by $\mathrm{UQ}$ in aeroelasticity are given in $[\underline{2}, \underline{4}, \underline{15}, \underline{46}]$. Applications to stability or optimization are described in $[\underline{1}, \underline{12}, \underline{31}, \underline{36}, \underline{38}, \underline{47}, \underline{62}]$ for example. In this paper the influence of operational and structural parametric uncertainties (inputs) on the deformation and aerodynamic performances (outputs) of a flexible wing in transonic regime, is quantified. This UQ analysis is performed thanks to polynomial surrogate models of the quantities of interest accounting for fluid-structure interactions. The air flow is solved by highfidelity computational fluid dynamics (CFD) tools [7, 21] while the wing motion and stresses are computed by a simplified beam model [39]. The coupling is ensured by balancing the aerodynamic loads for a prescribed lift force, considered as a variable input parameter, with the efforts in the wing for its induced shape. The overall procedure is outlined in [27, 39].

Polynomial surrogate of random functionals are typically obtained invoking the homogeneous, or polynomial chaos (PC) expansion introduced by Wiener [61] for stochastic processes. It is defined as the span of Hermite polynomial functionals of a Gaussian random variable. Mean-square con- 
vergence is guaranteed by the Cameron-Martin theorem [6]. It is optimal (i.e. exponential) for Gaussian processes but sub-optimal for non-Gaussian processes, as shown numerically in [63]. Generalized polynomial chaos (gPC) expansions with other families of polynomials which numerically recover the foregoing optimality property have then been studied in $[22, \underline{53}, \underline{63}]$. They consist in expanding a function of random variables into a linear combination of orthogonal polynomials with respect to the probability density functions (PDFs) of these underlying random variables. $\mathrm{PC}$ and $\mathrm{gPC}$ expansions are now widely used in engineering sciences as a constructive tool for representing random vectors, matrices, tensors or fields, and quantifying uncertainties in complex systems; see $[\underline{14}, \underline{24}, \underline{25}, \underline{28}, \underline{37}, \underline{43}, \underline{56}]$ and references therein. Applications to aeroelastic stability are considered in $[\underline{31}, \underline{38}, \underline{62}]$ for example.

Non-intrusive UQ techniques are typically considered in CFD, because the complex flow solvers are preferably treated as black boxes in order to compute the aerodynamic loads and other quantities of interest. Regarding non-intrusive PC and gPC expansions, two approaches for computing the polynomial coefficients of the output quantities of interest have usually been considered:

- the projection approach, in which they are computed by structured quadratures, i.e. Gauss quadratures, or unstructured quadratures, i.e. MonteCarlo or quasi Monte-Carlo sampling;

- the regression approach, minimizing some error measure or truncation tolerance of the polynomial expansion for some particular values of the inputs (which can be the quadrature sets invoked just above, for example). 
Both techniques suffer from the so-called "curse of dimensionality" when the dimension $D$ of the parameter space increases. Indeed, a polynomial expansion of total degree $q$ in $D$ variable parameters contains $P+1=\left(\begin{array}{c}q+D \\ q\end{array}\right) \approx \frac{D^{q}}{q !}$ coefficients for $D$ large. A direct way to compute them is to use a tensor product grid in the parameter space requiring about $N \approx\left\lfloor\frac{q}{2}\right\rfloor^{D}$ evaluations of the whole model, where $\lfloor\cdot\rfloor$ stands for the floor function. These $N$ runs are very often unaffordable for large parameter spaces and complex configurations, as in the present case dealing with three-dimensional, non-linear fluid-structure interactions. Smolyak's algorithm [52] introduces sparse grid quadratures involving $N \approx\left\lfloor\frac{q}{2}\right\rfloor^{\log D}$ points while preserving a satisfactory level of accuracy. In [49] it has been observed that such sparse rules typically become competitive with respect to tensor grids for dimensions $D \geq 4$. Consequently, collocation techniques with sparse quadratures or adaptive regression strategies have been developed in order to circumvent the dimensionality concern $[\underline{5}, \underline{28}, \underline{64}]$.

In this article we adopt the regression approach to propagate uncertainty in the aeroelastic computations. We also aim at benefiting from the sparsity of the processed outputs themselves (wing shape, drag and pitching moment coefficients) to reconstruct their $\mathrm{gPC}$ representations in a non-adaptive way [20]. Indeed, we rely on the observation that many cross-interactions between the input parameters are actually smoothened, or even negligible, once that have been propagated to some global quantities of interest processed from complex aerodynamic computations. The corresponding polynomial expansions should thus involve only low-order polynomials, such that the contribution of the higher-order polynomials is negligible. We can therefore expect 
to achieve a successful output recovery by the techniques known under the terminology of compressed sensing $[\underline{8}, \underline{19}]$. In this theory the reconstruction of a sparse signal on a given, known basis requires only a limited number of evaluations at randomly selected points-at least significantly less than the dimension $P$ of the basis. We thus resort to unstructured sampling sets to recover sparse outputs. Compressed sensing is formulated as a constrained, underdetermined system which may be solved by standard optimization algorithms.

The rest of the paper is organized as follows. In Section $\underline{2}$ the aeroelastic database for which surrogates are sought for is outlined. The aerodynamic model, the structural model, and the computational procedure for flow-structure interactions are introduced. The variable input parameters for this problem, and the sampling strategy for the computations are also detailed. Then Section 3 presents the construction of polynomial surrogates for the output quantities of interest identified in the foregoing section. Probability density functions and sensitivity indices are typically computed using those polynomials surrogates. Some conclusions are finally drawn in Section $\underline{4}$.

\section{Aeroelastic database}

In this section the numerical procedure and tools considered to generate the aeroelastic database to be used as a basis for uncertainty propagation, are outlined. The database consists of high-fidelity CFD-elastic simulations representative of an aircraft wing. The sampling of the aeroelastic input parameters covers a very large design space that enables to achieve significant 
aerodynamic output at cruise (the main output being the drag sensitivity to aerodynamic and structural parameters).

\subsection{Aeroelastic computation process}

The coupled fluid-structure problem is solved in terms of the conservative aerodynamic quantities $\boldsymbol{w}$ of the fluid flow, and the displacement $\boldsymbol{u}$ of the aircraft wing. They solve the non-linear Reynolds Average Navier-Stokes (RANS) equations and the linear beam equilibrium equations, respectively, written as the residual equations $\mathcal{R}_{\mathrm{f}}$ and $\mathcal{R}_{\mathrm{s}}$ for the fluid and the structure:

$$
\begin{aligned}
& \mathcal{R}_{\mathrm{f}}(\boldsymbol{w}, \mathcal{T}(\boldsymbol{u}) ; \boldsymbol{\xi})=0, \\
& \mathcal{R}_{\mathrm{s}}(\boldsymbol{u}, \mathcal{F}(\boldsymbol{w}) ; \boldsymbol{\xi})=0 .
\end{aligned}
$$

Here $\mathcal{F}(\boldsymbol{w})$ stands for the steady-state aerodynamic forces and moments acting on the wing through the fluid-structure interface $\Sigma_{\mathrm{fs}}, e . g$. the wing profile, as induced by fluid flow aerodynamic field $\boldsymbol{w}$, and $\mathcal{T}(\boldsymbol{u})$ is the position of that profile in the fluid flow induced by the structure motion $\boldsymbol{u}$. The latter as well as the associated strain tensor are assumed to remain small. At last, $\boldsymbol{\xi}$ stands for the variable aerodynamic and structural parameters considered in this study, namely the Mach number and the lift coefficient of the profile, and the bending and torsion stiffnesses of the wing at four control points; see Section 2.3 below for the description of the parameters ranges and associated database. In view of its non-linearity, the foregoing system is solved numerically by a staggered procedure, invoking a fixed-point argument. At the iteration step $n$, the aerodynamic field $\boldsymbol{w}^{(n)}$ is computed by the fluid flow solver for the position $\mathcal{T}^{(n)}\left(\boldsymbol{u}^{(n-1)}\right)$ of the interface $\Sigma_{\mathrm{fs}}$ induced by the wing motion $\boldsymbol{u}^{(n-1)}$ computed by the structure solver at the previous iteration 
step. In turn, the aerodynamic loads are updated to $\mathcal{F}^{(n)}\left(\boldsymbol{w}^{(n)}\right)$ which allows to compute the updated wing motion $\boldsymbol{u}^{(n)}$. This iterative scheme:

$$
\begin{gathered}
\mathcal{R}_{\mathrm{f}}\left(\boldsymbol{w}^{(n)}, \mathcal{T}^{(n)}\left(\boldsymbol{u}^{(n-1)}\right) ; \boldsymbol{\xi}\right)=0 \\
\mathcal{R}_{\mathrm{S}}\left(\boldsymbol{u}^{(n)}, \mathcal{F}^{(n)}\left(\boldsymbol{w}^{(n)}\right) ; \boldsymbol{\xi}\right)=0
\end{gathered}
$$

is repeated until convergence is reached in terms of the tolerance:

$$
\left|\boldsymbol{u}^{(n+1)}-\boldsymbol{u}^{(n)}\right| \leq \varepsilon_{\mathrm{s}}\left|\boldsymbol{u}^{(n)}\right|
$$

evaluated at the position of maximum deformations of the wing (typically the wing tip), and $\varepsilon_{\mathrm{s}} \ll 1$ is a small number. It starts from the jig shape $\boldsymbol{u}^{(0)}$ of the wing induced by gravity forces at ground level (see Fig. 5) before it is deformed further by the aerodynamic loads in cruise configuration. The same initial shape $\boldsymbol{u}^{(0)}$ is considered for all possible values of the parameters $\boldsymbol{\xi}$ in their ranges of variation, and the foregoing iterative process is subsequently run for all $\boldsymbol{\xi}$ 's of the database described in Section 2.3.

\subsubsection{Structure solver}

The aircraft wing is modeled as a rectilinear (or piecewise rectilinear) beam of which cross-sections $\Sigma(s)$ vary along the beam axis parameterized by the curvilinear coordinate $s \in \mathcal{C}=[0, L]$ where $L$ is the wingspan. The axis of the beam is constituted by its geometrical and inertial centers which are assumed to be superimposed, i.e. they form the neutral fiber. It is oriented by the unit vector $\boldsymbol{e}$ which together with the vertical axis $\hat{\boldsymbol{z}}:=\boldsymbol{e}_{1}$ and the bi-normal unit vector $\boldsymbol{e}_{2}=\boldsymbol{e} \times \boldsymbol{e}_{1}$ constitute a Frenet-Serret frame defined about $\mathcal{C}$; see Fig. 1 . This frame is fixed with respect to the moving frame attached to the aircraft travelling at the cruise Mach number $M$. Adopting a 
classical beam kinematical model, the displacement of any point $s=\left(s, \boldsymbol{s}_{\Sigma}\right)$ of the beam occupying the domain $\Omega_{\mathrm{s}}=\mathcal{C} \times \Sigma(s)$ of $\mathbb{R}^{3}$ reads:

$$
\boldsymbol{u}(\boldsymbol{s})=\boldsymbol{U}(s)+\boldsymbol{\theta}(s) \times \boldsymbol{s}_{\Sigma},
$$

where $\boldsymbol{s}=s \boldsymbol{e}+\boldsymbol{s}_{\Sigma}$, and $\boldsymbol{s}_{\Sigma} \perp \boldsymbol{e}$ is the position of $\boldsymbol{s} \in \Sigma(s)$ about the neutral fiber at $s . \boldsymbol{U}(s) \in \mathbb{R}^{3}$ is the displacement of the neutral fiber, and $\boldsymbol{\theta}(s) \in \mathbb{R}^{3}$ is the rotation vector of the cross-section $\Sigma(s)$ about the neutral fiber at $s$. The wing being fixed to the fuselage at $s=0$, they satisfy $\boldsymbol{U}(0)=\boldsymbol{\theta}(0)=\mathbf{0}$. Both the displacement and associated strain tensor are assumed to be small: $\|\boldsymbol{u}\| \ll L$ and $\left\|\nabla_{s} \otimes \boldsymbol{u}\right\| \ll 1$. Under Euler-Bernoulli kinematical assumption, the rigid cross-sections remain in addition perpendicular to the neutral fiber with the motion of the latter. This yields $\boldsymbol{\theta}(s)=\boldsymbol{e} \times \boldsymbol{U}^{\prime}(s)$ where $f^{\prime}=\frac{\mathrm{d} f}{\mathrm{~d} s}$ for any function $s \mapsto f(s)$. Also $\boldsymbol{a}_{\Sigma}:=(\boldsymbol{I}-\boldsymbol{e} \otimes \boldsymbol{e}) \boldsymbol{a}$ and $a_{e}=\boldsymbol{a} \cdot \boldsymbol{e}$ for any vector $\boldsymbol{a} \in \mathbb{R}^{3}$, where $\boldsymbol{I}$ is the identity matrix and $\boldsymbol{a} \otimes \boldsymbol{b}$ is the usual tensor product of vectors $\boldsymbol{a}$ and $\boldsymbol{b}$ such that for any $\boldsymbol{c},(\boldsymbol{a} \otimes \boldsymbol{b}) \boldsymbol{c}=(\boldsymbol{b} \cdot \boldsymbol{c}) \boldsymbol{a}$ with $\boldsymbol{b} \cdot \boldsymbol{c}$ the Euclidean scalar product of $\boldsymbol{b}$ and $\boldsymbol{c}$; we have in particular $\boldsymbol{s}_{\Sigma}=(\boldsymbol{I}-\boldsymbol{e} \otimes \boldsymbol{e}) \boldsymbol{s}$.

Now let $\mathcal{F}(\boldsymbol{w})=(\boldsymbol{f}(s), \boldsymbol{m}(s))$ by the linear aerodynamic forces and moments, respectively, exerted on the wing profile by the aerodynamic field $\boldsymbol{w}$. Then the beam displacement under Euler-Bernoulli kinematical assumption satisfies:

$$
\begin{array}{r}
E \boldsymbol{J}\left(\boldsymbol{e} \times \boldsymbol{U}_{\Sigma}^{\prime \prime \prime \prime}\right)-\boldsymbol{e} \times \boldsymbol{f}_{\Sigma}+\boldsymbol{m}_{\Sigma}^{\prime}=\mathbf{0}, \\
G j_{e} \theta_{e}^{\prime \prime}+m_{e}=0,
\end{array}
$$

for the flexural and torsional motions $\boldsymbol{U}_{\Sigma}$ and $\theta_{e}$, respectively. The axial motion $U_{e}$ of the beam along the direction of its neutral fiber is ignored. Here $E(s)$ is the Young's modulus of the wing at the cross-section $\Sigma(s), G(s)$ 


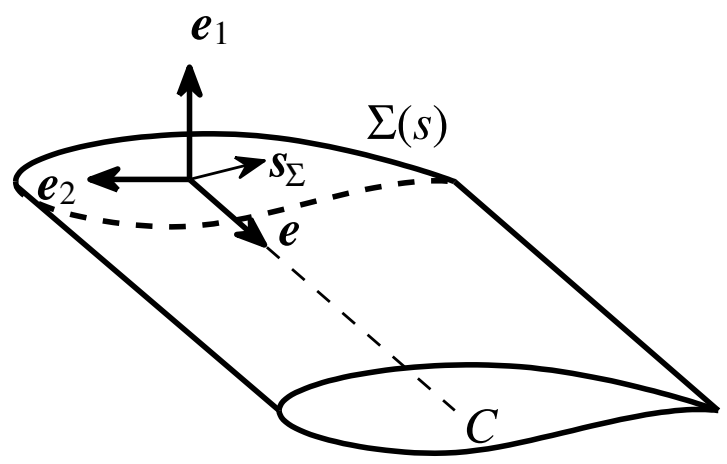

Figure 1: Wing beam model.

is its shear modulus, $\boldsymbol{J}(s)=\int_{\Sigma(s)}\left(\left\|\boldsymbol{s}_{\Sigma}\right\|^{2} \boldsymbol{I}-\boldsymbol{s}_{\Sigma} \otimes \boldsymbol{s}_{\Sigma}\right) \mathrm{d} \boldsymbol{s}_{\Sigma}$ is its tensor of area moments of inertia, and $j_{e}(s)=\boldsymbol{J}(s) \boldsymbol{e} \cdot \boldsymbol{e}$ is the polar moment of inertia with respect to its axis. The equations of motion (5) are solved in their weak form, applying the principle of virtual work and a finite element method whereby the neutral fiber is discretized into flexural/torsion beam elements. This procedure yields the residual $\mathcal{R}_{\mathrm{s}}$ in Eq. (2).

\subsubsection{Flow solver}

The aerodynamic loads $\mathcal{F}(\boldsymbol{w})$ are computed from the RANS equations for a Newtonian, compressible flow. Introducing the density $\varrho$, the momentum $\varrho \boldsymbol{v}$, and the total energy $\varrho E$ per unit of volume, the homogeneous NavierStokes equations for a compressible flow read:

$$
\begin{aligned}
\partial_{t} \varrho+\operatorname{div}(\varrho \boldsymbol{v}) & =0, \\
\partial_{t}(\varrho \boldsymbol{v})+\operatorname{Div}(\varrho \boldsymbol{v} \otimes \boldsymbol{v}-\boldsymbol{\sigma}) & =\mathbf{0}, \\
\partial_{t}(\varrho E)+\operatorname{div}[(\varrho E-\boldsymbol{\sigma}) \boldsymbol{v}+\boldsymbol{q}] & =0,
\end{aligned}
$$


where $\boldsymbol{\sigma}$ is the stress tensor and $\boldsymbol{q}$ is the heat flux vector due to thermal conductivity. For an isotropic Newtonian fluid, the stress tensor reads:

$$
\boldsymbol{\sigma}=-p \boldsymbol{I}+\boldsymbol{\tau}, \quad \boldsymbol{\tau}=\lambda \operatorname{div}(\boldsymbol{v}) \boldsymbol{I}+2 \mu \boldsymbol{D},
$$

where $p$ is the thermodynamic pressure, $\boldsymbol{D}$ is the strain rate tensor defined by $\boldsymbol{D}=\frac{1}{2}\left(\boldsymbol{\nabla} \otimes \boldsymbol{v}+\boldsymbol{\nabla} \otimes \boldsymbol{v}^{\top}\right), \boldsymbol{A}^{\top}$ stands for the transpose of a matrix $\boldsymbol{A}$, and $\lambda$ and $\mu$ are the bulk and dynamic viscosities of the fluid. For a perfect gas with constant specific heats of ratio $\gamma=c_{p} / c_{v}$, the internal energy $e$ and pressure $p$ are given by the equations of state:

$$
e(T)=c_{v} T=E-\frac{1}{2}\|\boldsymbol{v}\|^{2}, \quad p(\varrho, T)=\varrho R T,
$$

where $T$ is the temperature, and $R=c_{p}-c_{v}$ is the specific gas constant.

In order to erase the fine structures of the turbulent fields which are typically not accessible to measurements or simulations, the turbulent quantity $\phi$ is split into a mean value $\bar{\phi}$ and a fluctuating value $\delta \phi$ as:

$$
\phi=\frac{\overline{\varrho \phi}}{\bar{\varrho}}+\delta \phi,
$$

where $\widetilde{\phi}:=\overline{\varrho \phi} / \varrho$ is the so-called mass-weighted Favre average. In the context of steady flows, and invoking an ergodicity principle, the mean values above are interpreted as either (i) a time average over a time greater than the characteristic time scale of turbulence but smaller than the characteristic period for the time evolution of the macroscopic properties of the flow; or (ii) as an ensemble average over different possible realizations of the fine turbulent structures. Now let $\boldsymbol{w}=\bar{\varrho}(1, \widetilde{\boldsymbol{v}}, \widetilde{E}+\widetilde{k})$ be the vector of the conservative aerodynamic variables, where $k=\frac{1}{2}\|\delta \boldsymbol{v}\|^{2}$ is the kinetic energy of 
the turbulent motion. Also let $\Omega$ be the fixed computational domain (or any cell of the mesh) of outward unit normal $\hat{\boldsymbol{n}}$. Then applying this averaging operator (7) to the Navier-Stokes equations (6) and integrating over $\Omega$ yields the RANS equations in their integral form:

$$
\frac{\mathrm{d}}{\mathrm{d} t} \int_{\Omega} \boldsymbol{w} \mathrm{d} \Omega+\int_{\partial \Omega} \boldsymbol{F}_{c}(\boldsymbol{w}) \cdot \hat{\boldsymbol{n}} \mathrm{d} S=\int_{\partial \Omega} \boldsymbol{F}_{d}(\boldsymbol{w}, \boldsymbol{\nabla} \boldsymbol{w}) \cdot \hat{\boldsymbol{n}} \mathrm{d} S,
$$

where $\boldsymbol{F}_{c}$ and $\boldsymbol{F}_{d}$ are the convective and diffusive fluxes given by:

$$
\begin{aligned}
\boldsymbol{F}_{c}(\boldsymbol{w}) & =\bar{\varrho}\left[\begin{array}{c}
\widetilde{\boldsymbol{v}} \\
\widetilde{\boldsymbol{v}} \otimes \widetilde{\boldsymbol{v}}+\left(\frac{\bar{p}}{\overline{\underline{p}}}+\frac{2}{3} \widetilde{k}\right) \boldsymbol{I} \\
\left(\widetilde{E}+\frac{\bar{p}}{\overline{\underline{\rho}}}+\frac{5}{3} \widetilde{k}\right) \widetilde{\boldsymbol{v}}
\end{array}\right], \\
\boldsymbol{F}_{d}(\boldsymbol{w}, \boldsymbol{\nabla} \boldsymbol{w}) & =\left[\begin{array}{c}
\mathbf{0} \\
\left(\lambda-\frac{2}{3} \mu_{t}\right) \operatorname{div}(\widetilde{\boldsymbol{v}}) \boldsymbol{I}+2\left(\mu+\mu_{t}\right) \widetilde{\boldsymbol{D}} \\
\left(\lambda-\frac{2}{3} \mu_{t}\right) \operatorname{div}(\widetilde{\boldsymbol{v}}) \widetilde{\boldsymbol{v}}+2\left(\mu+\mu_{t}\right) \widetilde{\boldsymbol{D}} \widetilde{\boldsymbol{v}}-\overline{\boldsymbol{q}}-\overline{\boldsymbol{q}}_{t}
\end{array}\right] .
\end{aligned}
$$

This model introduces the eddy (turbulent) viscosity parameter $\mu_{t}$ and Prandtl number $\mathrm{Pr}_{t}$ such that, within Boussinesq's approximation, the Reynolds stress tensor $\boldsymbol{\tau}_{t}=-\varrho \delta \boldsymbol{v} \otimes \delta \boldsymbol{v}$ reads after averaging:

$$
\overline{\boldsymbol{\tau}}_{t}=-\frac{2}{3}\left(\bar{\varrho} \widetilde{k}+\mu_{t} \operatorname{div} \widetilde{\boldsymbol{v}}\right) \boldsymbol{I}+2 \mu_{t} \widetilde{\boldsymbol{D}},
$$

and the enthalpy turbulent diffusion flux $\boldsymbol{q}_{t}=\varrho \delta h \delta \boldsymbol{v}$, with the specific enthalpy $h=e+p / \varrho$, reads after averaging:

$$
\overline{\boldsymbol{q}}_{t}=-\frac{\gamma \mu_{t}}{P r_{t}} \nabla \widetilde{e} .
$$

These quantities have to be specified in order to close the RANS system (8) above. In the Spalart-Allmaras turbulence model closure [54] used in the subsequent analyses, the turbulent kinetic energy $k$ is negligible, the 
turbulent Prandtl number is fixed (typically $P r_{t}=0.9$ ), and the turbulent viscosity $\mu_{t}$ is the solution of a scalar transport equation. The latter is solved in parallel with the RANS system (8), since it has the same form in its integrated version (with an additional source term though). We refer to [54] for further details. This model has been used for a wide range of flows for which the comparisons with experimental data have been satisfactory; see e.g. $[\underline{7}, \underline{21}]$ and references therein for some complex applications.

At first, Eq. (8) is discretized in space by a cell-centered finite-volume method, yielding the residual $\mathcal{R}_{\mathrm{f}}$ in Eq. (2). The convective flux is computed using the Jameson centered scheme [32] with artificial viscosity $\left(\chi_{2}=0.5\right.$, $\left.\chi_{4}=0.016\right)$ outside of the boundary layers. The diffusive flux is computed as the half sum of the normal flux densities in the two adjacent cells sharing the current interface (with due care of the boundary conditions on external interfaces), considering corrected cell-centered gradients of the fluid velocity in these cells. Each cell-centered velocity gradient is computed as the average of $\widetilde{\boldsymbol{v}} \otimes \hat{\boldsymbol{n}}$ over the surrounding interfaces invoking Green-Ostrogradski's formula, and then the gradient at the center of the current interface is obtained by considering the control volume centered on that interface and composed of the two half cells neighboring it. The diffusive flux of the transport equation for the turbulent variable is discretized using a similar approach, whereas a first order Roe flux is used for the convective term. Finally, the source term of the transport equation is computed using the temperature gradients at the center of the cells. Secondly, the semi-discretized version of Eq. (8) is solved in time using a backward Euler scheme up to the convergence towards a steady-state solution. The linearization of the resulting non-linear 
implicit system of equations is performed using the Lower-Upper Symmetric Successive Over-relaxation (LU-SSOR) scheme [65] with four relaxation cycles. The convergence is accelerated by the use of multigrid techniques for steady flows. A uniform flow is considered as the initial conditions for the iterations with respect to the time parameter.

Once the steady-sate solution $\boldsymbol{w}$ has been computed, the aerodynamic loads exerted on the wing profile are given by:

$$
\begin{aligned}
\boldsymbol{f}(s) & =\int_{\Sigma(s) \cap \Sigma_{\mathrm{fs}}}\left(\bar{p}-p_{\infty}\right) \hat{\boldsymbol{n}} \mathrm{d} l+\int_{\Sigma(s) \cap \Sigma_{\mathrm{fs}}}\left(\overline{\boldsymbol{\tau}}+\overline{\boldsymbol{\tau}}_{t}\right) \hat{\boldsymbol{n}} \mathrm{d} l, \\
\boldsymbol{m}(s) & =\int_{\Sigma(s) \cap \Sigma_{\mathrm{fs}}} \boldsymbol{s}_{\Sigma} \times\left(\bar{p}-p_{\infty}\right) \hat{\boldsymbol{n}} \mathrm{d} l+\int_{\Sigma(s) \cap \Sigma_{\mathrm{fs}}} \boldsymbol{s}_{\Sigma} \times\left(\overline{\boldsymbol{\tau}}+\overline{\boldsymbol{\tau}}_{t}\right) \hat{\boldsymbol{n}} \mathrm{d} l,
\end{aligned}
$$

where $\Sigma(s) \cap \Sigma_{\mathrm{fs}}$ is the wing-flow interface at the position $s$ spanwise (i.e. the boundary of $\Sigma(s)$ ), and $\hat{\boldsymbol{n}}$ is its outward unit normal. Also $p_{\infty}$ is the pressure on the upstream boundary of the computational domain.

\subsubsection{Coupling the solvers}

Various techniques have been derived to couple the fluid and structure solvers used in a staggered process, depending on the numerical schemes they use; see e.g. [39] and references therein. Two steps are required: (i) the derivation of the aerodynamic loads $\mathcal{F}(\boldsymbol{w})$ to be imposed to the wing beam model, and (ii) the application of the wing profile motion $\mathcal{T}(\boldsymbol{u})$ to the

fluid mesh to update the flow computation. Regarding the first step, since the fluid mesh and the beam mesh do not coincide in most situations, and the fluid mesh is typically much finer than the structure mesh, a dedicated approach has to be implemented. In this study, an influence area (defined by the half-distance to the two neighboring nodes) is attached to each node of the structure mesh, and the contribution of the aerodynamic pressure 
and viscous forces of all cells of the fluid mesh intersecting that influence area is added to form the surface forces and moments applied to each node. Regarding the second step, once the structure motion $\boldsymbol{U}(s)$ and $\boldsymbol{\theta}(s)$ have been computed, they are transferred to the fluid mesh using the kinematical relation (4) for all nodes located at a given distance, say $R$, of the structure mesh. That is, for a node $\boldsymbol{x}^{j}=\left(s^{j}, \boldsymbol{x}_{\Sigma}^{j}\right)$ of the fluid mesh located in a cylinder of radius $R$ about the wing, its new coordinates in the deformed mesh $\mathcal{T}(\boldsymbol{u})$ are updated to $\boldsymbol{x}^{j}+\boldsymbol{U}\left(s^{j}\right)+\boldsymbol{\theta}(s) \times \boldsymbol{x}_{\Sigma}^{j}$. This updating is gradually damped out outside the cylinder of radius $R$ to finally be nullified at the distance $a R$, $a>1$, about the wing. Typically $R$ is chosen as twice the local chord length streamwise, and $a=5$. This approach is justified inasmuch as only small displacement and strains are considered for the wing, and the beam mesh is kept unchanged for all the coupling process. The foregoing two steps are described in detail in [39]. They have been implemented in ONERA's CFD solver elsA $[\underline{7}, \underline{21}, \underline{39}, \underline{41}]$, which has been used for this research.

\subsection{Aeroelastic case: ALBATROS wing}

The configuration of interest is the ALBATROS model proposed in [11]; see Fig. 2. It has been designed at ONERA in an internal project between 2010 and 2013. The ALBATROS acronym can be translated into "laminar strut braced wing with reduced drag by multidisciplinary optimization" (Aile Laminaire hauBAnée à Traînée Réduite par Optimisation multidiSciplinaire, in French). The purpose of this project was to design and investigate the pros and cons of a strut braced wing configuration, with high-fidelity analyses in both the structural response and the aerodynamic loads. The aircraft has been designed for medium range $(3,000 \mathrm{Nm})$ with 180 passengers (equivalent 


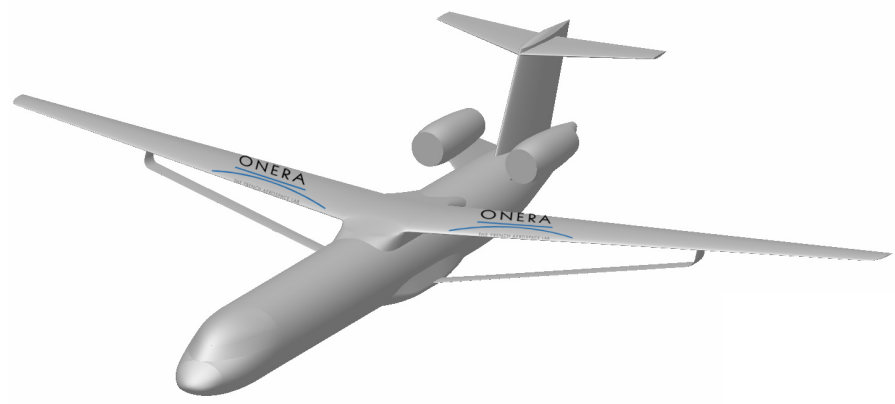

Figure 2: ALBATROS configuration.

to an Airbus A321). To achieve natural laminar flow on the wing, the wing sweep is limited to $16^{\circ}$ (at 25\% of the chord) and thus the design cruise Mach number is $\underline{M}=0.75$. The design lift coefficient had been set to $\underline{C}_{L}=0.60$. These nominal parameters are underlined to stress the difference with their actual, yet unknown values $M$ and $C_{L}$; see the subsequent section.

\subsubsection{Aerodynamic model}

For the purpose of the present study, the ALBATROS configuration has been simplified. The strut and tail elements have been removed so that only the wing and fuselage are accounted for; see Fig. $\underline{3}$. Since the geometry and loads are symmetric with respect to the vertical plan parallel to the streamflow, only one half of the numerical model is actually solved. Another simplification has been to consider only turbulent flow instead of laminar flow. This choice was made to simplify the simulations because the laminar/turbulent transition would have induced additional non-linearity to the database. The operational aerodynamic parameters considered as uncertain for the database are the cruise Mach number $M$ and lift coefficient $C_{L}$, 
though their variations are kept within an acceptable range for a cruise (see Section 2.3 below).

The aerodynamic mesh is structured and fully coincident. It consists of 9,008,512 cells and 9,879,020 nodes. For parallelism purposes, the mesh is decomposed into 240 blocks displayed over 60 processors. An isotropic multigrid acceleration technique (for a total of 3 grid levels, each time keeping one node over 2) is implemented. The Courant-Friedrichs-Lewy (CFL) stability condition CFL $=50$ is chosen for the backward Euler time integration scheme. The aerodynamic simulations are post-processed to derive a detailed phenomenological drag breakdown through the far-field drag analysis outlined in $[17,18]$ (and implemented in ONERA's ffd01 software). This post-processing allows first to convert the pressure/friction drag breakdown given by the CFD solvers into a physical breakdown: lift induced drag $C_{D \text {,lift induced, viscous drag }} C_{D \text {,viscous pressure }}+C_{D \text {,friction }}$, and wave drag $C_{D \text {,wave }}$. The cells containing wave or viscous drag are isolated to allow for specific volume integration of drag; see Fig. 4 . This analysis also enables to remove all numerical spurious contributions $C_{D \text {,spurious }}$ that stem from the imperfect numerical resolution of the gradients-at leading edges for instance. It is important to isolate this contribution because the mesh is deformed to represent various wing shapes, and thus this contribution evolves from one shape to another. Using this analysis increases the precision of the aeroelastic database; see [30]. The output of the database contains the drag breakdown for each parameter set, as well as the pitching moment coefficient $C_{m}$. The overall 


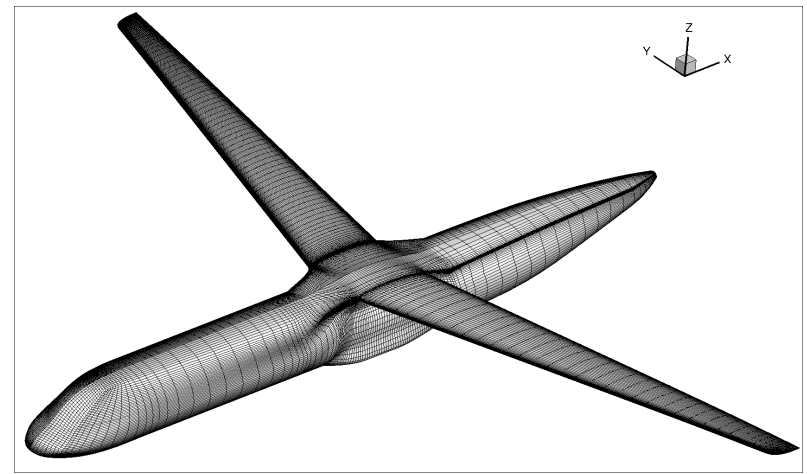

Figure 3: Aerodynamic surface mesh (mix of the full grid and the 1 node over 2 grid).

lift, drag, and pitching moment coefficients are defined by:

$$
\begin{aligned}
C_{L} & =\frac{\left(\int_{\Sigma_{\mathrm{fs}}}\left(p-p_{\infty}+\overline{\boldsymbol{\tau}}+\overline{\boldsymbol{\tau}}_{t}\right) \hat{\boldsymbol{n}} \mathrm{d} \boldsymbol{s}_{\Sigma}\right) \cdot(\hat{\boldsymbol{\alpha}} \times \hat{\boldsymbol{y}})}{\frac{1}{2} \gamma p_{\infty} S M^{2}}, \\
C_{D} & =\frac{\left(\int_{\Sigma_{\mathrm{fs}}}\left(p-p_{\infty}+\overline{\boldsymbol{\tau}}+\overline{\boldsymbol{\tau}}_{t}\right) \hat{\boldsymbol{n}} \mathrm{d} \boldsymbol{s}_{\Sigma}\right) \cdot \hat{\boldsymbol{\alpha}}}{\frac{1}{2} \gamma p_{\infty} S M^{2}}, \\
C_{m} & =\frac{\left(\int_{\Sigma_{\mathrm{fs}}} \boldsymbol{s}_{\Sigma} \times\left(p-p_{\infty}+\overline{\boldsymbol{\tau}}+\overline{\boldsymbol{\tau}}_{t}\right) \hat{\boldsymbol{n}} \mathrm{d} \boldsymbol{s}_{\Sigma}\right) \cdot \hat{\boldsymbol{y}}}{\frac{1}{2} \gamma c p_{\infty} S M^{2}},
\end{aligned}
$$

respectively, where $S$ is the reference surface of the wing, $c$ is the reference chord length, and $\hat{\boldsymbol{\alpha}}$ is the upstream flow direction. In the fixed reference frame $(\hat{\boldsymbol{x}}, \hat{\boldsymbol{y}}, \hat{\boldsymbol{z}})$ attached to the aircraft such that, say, $\hat{\boldsymbol{x}}$ is parallel to the axis of the aircraft and $\hat{\boldsymbol{z}}$ is the upward direction (see Fig. 3), the angle of attack $\alpha$ is $\cos \alpha=\hat{\boldsymbol{\alpha}} \cdot \hat{\boldsymbol{x}}$.

\subsubsection{Structure model}

A simplified, equivalent beam model has been created for the purpose of this study. It is derived following the work done in [27] on aero-structure optimization (InAirSsi module [26]). The jig shape $\boldsymbol{u}^{(0)}$ has been derived 


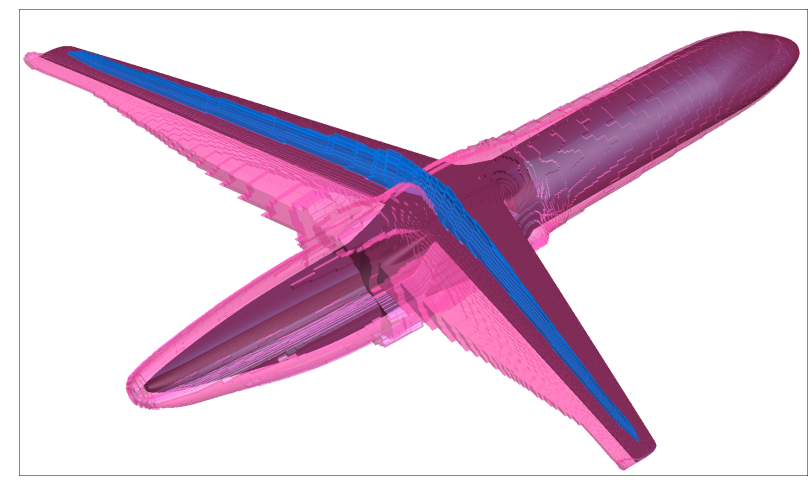

Figure 4: Far-field drag analysis $[17,18]$. The wave drag volume is in blue and the viscous pressure drag volume is in pink.

from a $1 \mathrm{~g}$ loading on the beam structure; see Fig. $\underline{5}$. It is kept fixed for all the different shapes (structure parameters) investigated in this study, as already mentioned in Section 2.1. The meaning of this is that the shape to be manufactured is designed once and for all and any structure uncertainty will be visible on the flight shape, which is a relevant scheme for an aircraft process. If the jig shape had been adapted for each structure parameter, the uncertainty on cruise deformation and drag would only come from the jig determination process and would be hardly noticeable. The aeroelastic simulations are carried out starting from the $1 \mathrm{~g}$ shape to accelerate the convergence (accounting for the jig to $1 \mathrm{~g}$ efforts and moments). The structure variable design parameters are directly related to the torsion and bending stiffnesses. Four fixed locations are chosen spanwise and the design parameters consist of coefficients to be applied to the bending stiffnesses $E \boldsymbol{J}$ and torsion stiffness $G j_{e}$ in Eq. (5); see Fig. $\underline{6}$. Large deviations are achieved on the flight shape for different stiffness parameters, as seen on Fig. $\underline{7}$. Since only one half of the numerical model is considered, these variabilities are 
applied to a single wing. Future works shall consider different parameters for both wings and the influence of such a dissymmetry on the aerodynamic coefficients, for example.

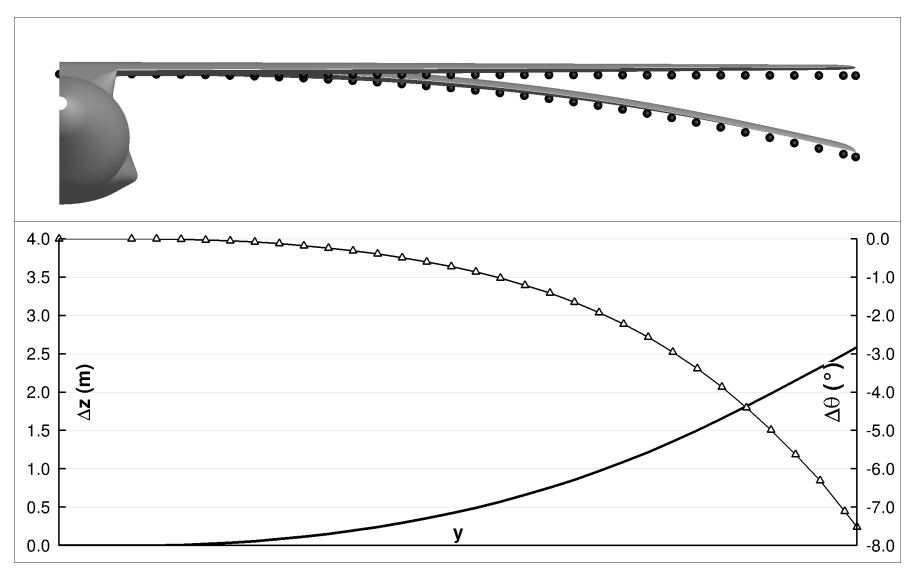

Figure 5: Structure modes of the beam and jig to $1 \mathrm{~g}$ loads: deflection (solid line) and torsion $(\triangle$ line $)$.

\subsubsection{Aeroelastic equilibrium for imposed lift}

For each set of input parameters, including the lift coefficient, the wing shape must be at equilibrium for the corresponding lift force. The balancing process is sketched on Fig. 8 . Two loops are nested:

- The outer loop concerns the prescribed lift: the angle of attack $\alpha$ is varied to reach the prescribed lift coefficient.

- The inner loop concerns the aeroelastic computation itself: for a given angle of attack $\alpha$, the wing shape corresponding to the equilibrium of the aerodynamic loading and the structural response is sought for through an iterative process. For each iteration, $300 \mathrm{CFD}$ cycles are computed. The integrated efforts and moments are transferred to the beam according to 


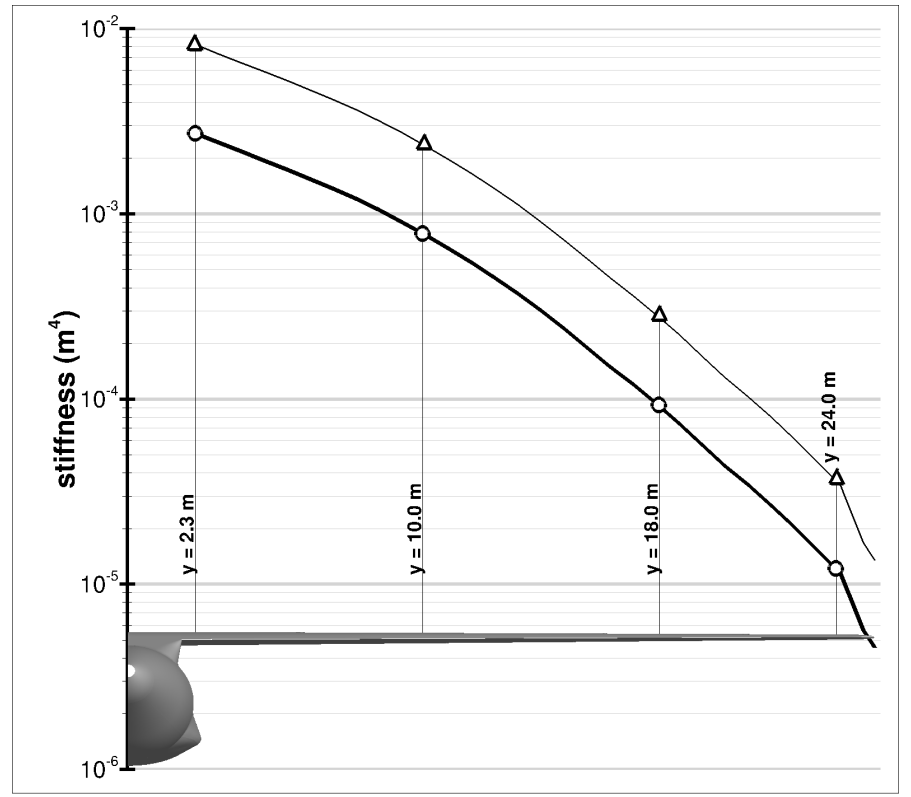

Figure 6: Bending (o) and polar $(\Delta)$ moments of inertia of the wing in the reference configuration. The vertical lines indicate the location of the control points/cross-sections.

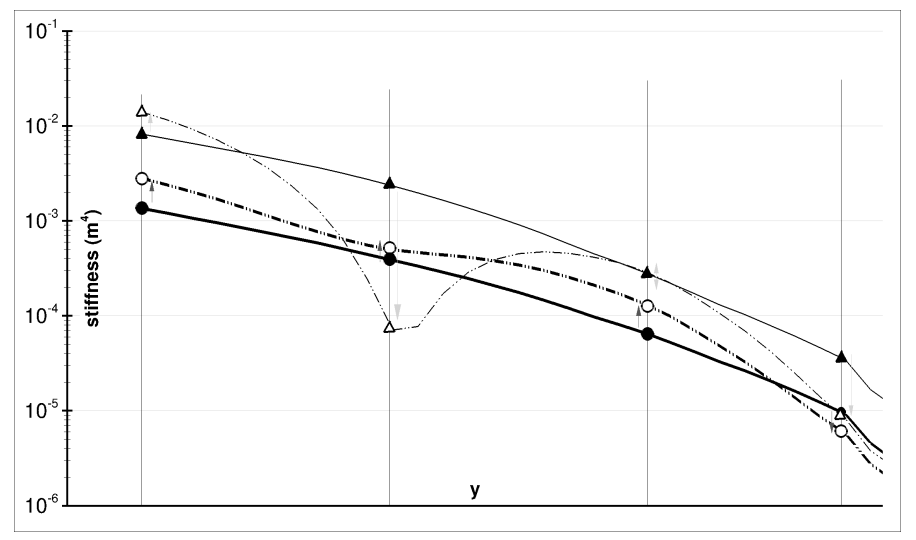

Figure 7: Example of two different wing shapes obtained with two different sets of bending (o) and torsion $(\Delta)$ stiffness parameters. The vertical lines indicate the location of the control points/cross-sections. 


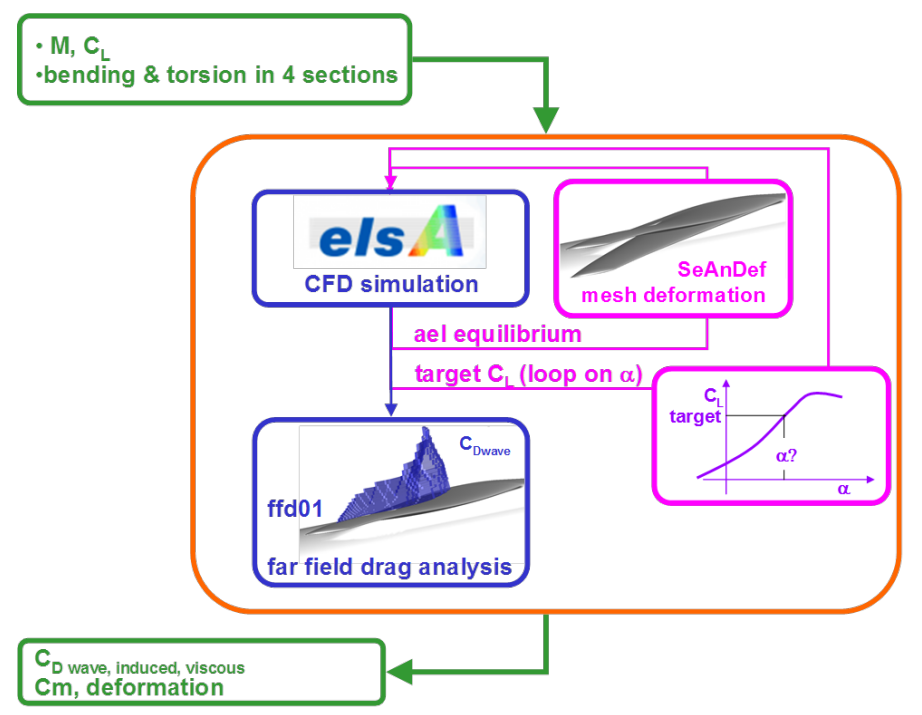

Figure 8: Aeroelastic process for each set of parameters.

the methodology outlined in Section 2.1.3, and the corresponding beam displacements are computed using a dedicated module of the els $A$ software $[\underline{7}, \underline{21}, \underline{39}]$. The induced aerodynamic mesh deformations are applied thanks to another dedicated analytical tool (SeAnDef) described in [41]. When convergence is reached (for a tolerance $\varepsilon_{\mathrm{s}}$ given here as $\varepsilon_{\mathrm{s}}=10^{-2}$ in Eq. (3) for the wing tip bend and twist defined in Section 2.3.3), the solution is analyzed with the far-field drag breakdown of $[17,18]$ using the ffd01 software. The whole process is driven by a python script and requires about 6 hours (wall-clock time) on 60 cores for each set of parameters $\boldsymbol{\xi}$.

\subsection{ALBATROS database}

\subsubsection{Manual range quantification}

Prior to the high-fidelity computations, the parameter variation amplitudes have been checked through 19 simulations, considering only one varia- 
tion at a time. The results are gathered in the Table 1 below. The sensitivity of wing deformation and thus of drag is far more pronounced for the bending than for the torsion. Therefore larger multiplication factors are applied to torsion to detect effects on the outputs (drag). However, when reaching too high or too low values, the aeroelastic simulations diverge. The resulting ranges for the 10 parameters have thus been identified as:

- Mach number $M \equiv \xi_{1}$ with $\xi_{1} \in[0.74,0.76]$;

- lift coefficient $C_{L} \equiv \xi_{2}$ with $\xi_{2} \in[0.55,0.65]$;

- 4 bending parameters $\xi_{3}, \xi_{4}, \xi_{5}$, and $\xi_{6}$ in the range $[0.5,2.0]$ which are multiplied to the reference bending stiffnesses $E \boldsymbol{J}$ in each control crosssection (see Fig. $\underline{6}$ );

- 4 torsion parameters $\xi_{7}, \xi_{8}, \xi_{9}$, and $\xi_{10}$ in the range $[0.02,10.0]$ which are multiplied to the reference torsion stiffness $G j_{e}$ in each control crosssection (see Fig. $\underline{6}$ ).

\subsubsection{Latin Hypercube Sampling}

A Latin Hypercube Sampling (LHS) has been set on the parameter ranges determined manually (optimized for space filling [3]). 100 samples have been generated to allow for a reasonable CPU time and to reach the empirical target of 10 samples per parameters. The minimum torsion value proved to be too aggressive and led to diverging wing shapes when associated with some other parameters. Thus, several sets of parameters did not lead to converged solutions (loss of 17 points). In the end, by combining the manual samples and the LHS samples, 102 points have been completely evaluated (about 10 points per parameter). The sampling for the various parameters 


\begin{tabular}{|c||c|c|c|}
\hline & $\boldsymbol{\xi}$ & $C_{D, \text { far-field }}$ & twist $\varphi\left(^{\circ}\right)$ \\
\hline \hline$M$ & 0.745 & $216 \mathrm{e}-04$ & -7.1 \\
$C_{L}$ & 0.55 & $203 \mathrm{e}-04$ & -6.8 \\
bending & $0.5 \times E \boldsymbol{J}$ & $226 \mathrm{e}-04$ & -10.0 \\
torsion & $0.1 \times G j_{e}$ & $218 \mathrm{e}-04$ & -7.2 \\
\hline
\end{tabular}

\begin{tabular}{|c||c|c|c|}
\hline & $\boldsymbol{\xi}$ & $C_{D, \text { far-field }}$ & twist $\varphi\left(^{\circ}\right)$ \\
\hline \hline$M$ & 0.750 & $219 \mathrm{e}-04$ & -7.2 \\
$C_{L}$ & 0.60 & $219 \mathrm{e}-04$ & -7.2 \\
bending & $1.0 \times E \boldsymbol{J}$ & $219 \mathrm{e}-04$ & -7.2 \\
torsion & $1.0 \times G j_{e}$ & $219 \mathrm{e}-04$ & -7.2 \\
\hline
\end{tabular}

\begin{tabular}{|c||c|c|c|}
\hline & $\boldsymbol{\xi}$ & $C_{D, \text { far-field }}$ & twist $\varphi\left(^{\circ}\right)$ \\
\hline \hline$M$ & 0.755 & $224 \mathrm{e}-04$ & -7.3 \\
$C_{L}$ & 0.65 & $238 \mathrm{e}-04$ & -7.6 \\
bending & $2.0 \times E \boldsymbol{J}$ & $222 \mathrm{e}-04$ & -4.7 \\
torsion & $10.0 \times G j_{e}$ & $218 \mathrm{e}-04$ & -7.2 \\
\hline
\end{tabular}

Table 1: Manual parameter sensitivity. The middle table is for nominal parameters.

is presented in Fig. 10. The original manual sampling is included (red dots displayed as a cross over the parametric space). The other symbols represent the LHS sampling (the converged samples are in red and the other ones are in blue).

\subsubsection{Outputs}

The output quantities of interest are: 
- the angle of attack $\alpha$;

- the CFD code drag outputs: pressure-induced contribution $C_{D \text {,pressure }}$ and friction-induced contribution $C_{D \text {,friction }}$;

- the drag coefficients obtained from a far-field drag analysis: lift-induced

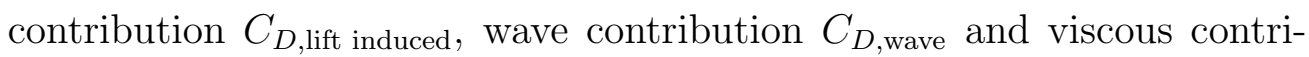
bution $C_{D \text {,viscous pressure }}$ (which adds to the friction-induced contribution $\left.C_{D \text {,friction }}\right)$;

- the numerical spurious drag contribution $C_{D \text {,spurious; }}$;

- the CFD code pitching moment coefficient $C_{m}$;

- the wing shape characterized by the wing tip bend $U=U_{z}(L)$ and maximum twist $\varphi$, defined as the relative differential vertical displacement between the leading edge $\boldsymbol{L}$ and trailing edge $\boldsymbol{T}$ aligned in the direction $\hat{\boldsymbol{x}}$ of the streamflow (see Fig. 9); namely:

$$
\begin{aligned}
\varphi & =\max _{0 \leq s \leq L} \tan ^{-1}\left(\frac{\boldsymbol{u}(\boldsymbol{L}(s))-\boldsymbol{u}(\boldsymbol{T}(s))}{\|\boldsymbol{L}(s)-\boldsymbol{T}(s)\|} \cdot \hat{\boldsymbol{z}}\right) \\
& =\max _{0 \leq s \leq L} \tan ^{-1}\left(\frac{U_{z}\left(s_{L}\right)-U_{z}\left(s_{T}\right)}{c_{L}(s)+c_{T}(s)}-\cos \phi \frac{\theta_{e}\left(s_{L}\right) c_{L}(s)+\theta_{e}\left(s_{T}\right) c_{T}(s)}{c_{L}(s)+c_{T}(s)}\right) \\
& \simeq \max _{0 \leq s \leq L} \tan ^{-1}\left(\frac{U_{z}\left(s_{L}\right)-U_{z}\left(s_{T}\right)}{c_{L}(s)+c_{T}(s)}-\theta_{e}(s) \cos \phi\right),
\end{aligned}
$$

where $\cos \phi=|\boldsymbol{e} \cdot \hat{\boldsymbol{y}}|$, and $s_{L}$ and $s_{T}$ are the curvilinear coordinates of the leading edge and trailing edge, respectively, on the neutral fiber such that $\boldsymbol{L}(s)=s_{L} \boldsymbol{e}+c_{L}(s) \cos \phi \boldsymbol{e}_{2}$ and $\boldsymbol{T}(s)=s_{T} \boldsymbol{e}-c_{T}(s) \cos \phi \boldsymbol{e}_{2}$, and $\|\boldsymbol{L}(s)-\boldsymbol{T}(s)\|=c_{L}(s)+c_{T}(s)$ is the local cord length streamwise. The last approximate equality stems from $\theta_{e}\left(s_{L}\right) \simeq \theta_{e}\left(s_{T}\right) \simeq \theta_{e}(s)$ where the twist is maximum. The relevance of this quantity in aeroelastic analysis is further discussed in e.g. [30]. 


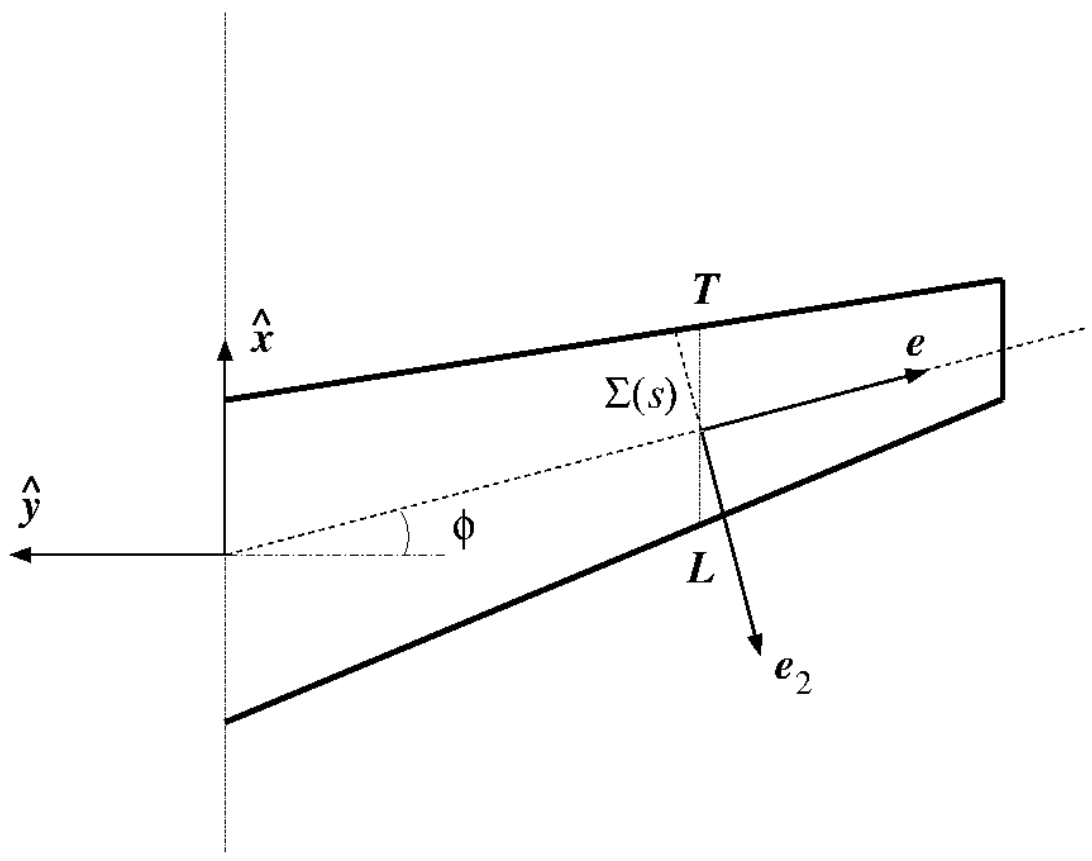

Figure 9: Geometrical setting for the definition of the wing twist.

The deformed wing shape, and the pressure and friction fields on each configuration are also post-processed.

\subsubsection{Brief analysis of the results}

Some input-output and output-output dependencies have been plotted on Fig. 11 through Fig. 14. Fig. 11 presents various dependencies between the input parameters and the aeroelastic outputs. If some dependency can be drawn between the drag and the Mach number or lift coefficient, no simple explicit relationship can be derived with the bending and torsion parameters presented here. On the contrary, some obvious dependencies with the input can be extracted for some specific outputs (see Fig. 12 for aerodynamics and Fig. 13 for aeroelasticity). Some dependencies can be simple (well-known 
correlation-linear dependence-between lift induced drag and lift, ${ }_{-}^{1}$ spurious drag as a function of Mach number, bending at the tip of the wing as a function of the bending parameter in the second section). Some other outputs exhibit strong trends but depend on multiple parameters. Finally, some cross dependencies between outputs are highlighted on Fig. 14. A high correlation can be denoted between wave drag and viscous pressure drag (top left plot). A less trivial near-correlation exists between the tip twist and the tip bend and other complex dependencies are also presented. This database contains many input parameters and outputs (aerodynamics and steady aeroelasticity). Precise computations enable to extract well-known or subtle dependencies between the parameters for a flexible wing in transonic regime. These idiosyncrasies make it a highly valuable database on which to propagate uncertainties.

\section{Uncertainty propagation}

The computation of the marginal PDFs and first moments (mean, standard deviation, skewness, kurtosis...) of the output quantities of interest when the variability of the parameters above (see Section 2.3.3) is accounted for, is done thank to surrogate models, or response surfaces. We more particularly focus on polynomial surrogates in this study.

\footnotetext{
${ }^{1}$ It should be noted that the strong dependence evidenced by the top right Fig. 12 is actually quadratic, and not linear.
} 

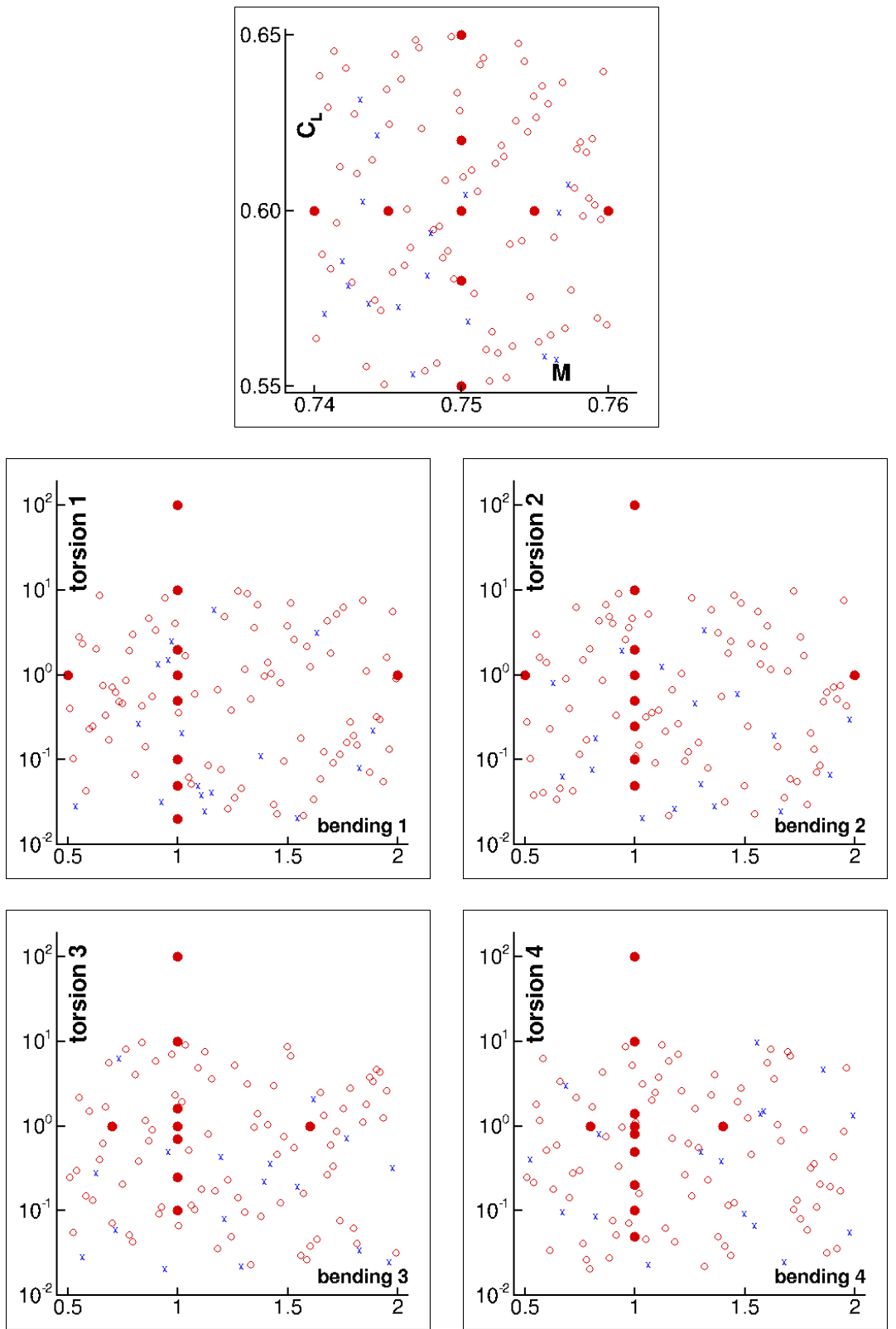

Figure 10: Latin Hypercube Sampling (LHS) set. Red dots $(\bullet)$ correspond to the original manual sampling, red circles (०) are the other successfully computed (converged) points of the LHS set, and the blue crosses $(x)$ are the points that did not lead to convergence. 

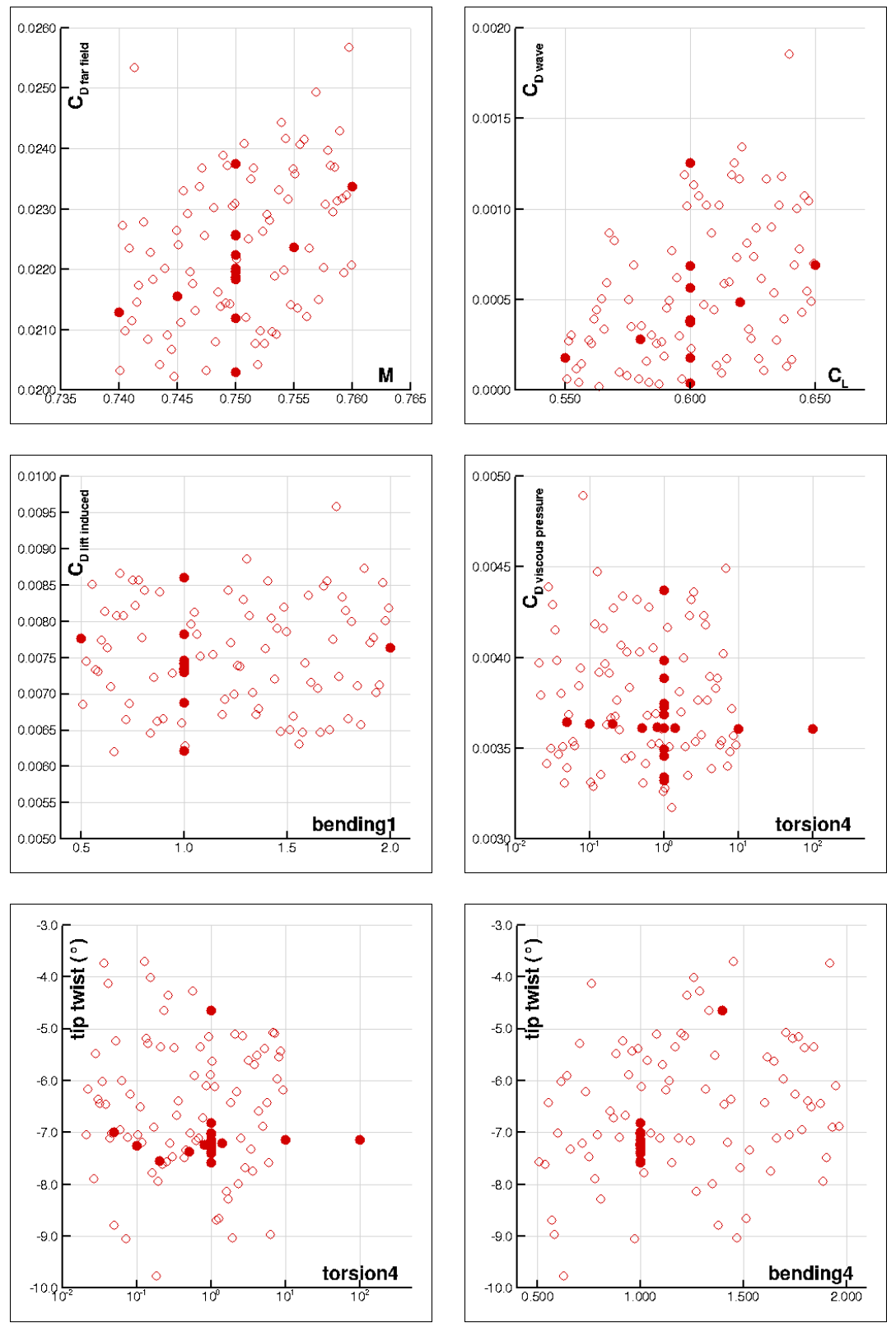

Figure 11: Various input-output dependencies (• manual sampling set, $\circ$ LHS set). 

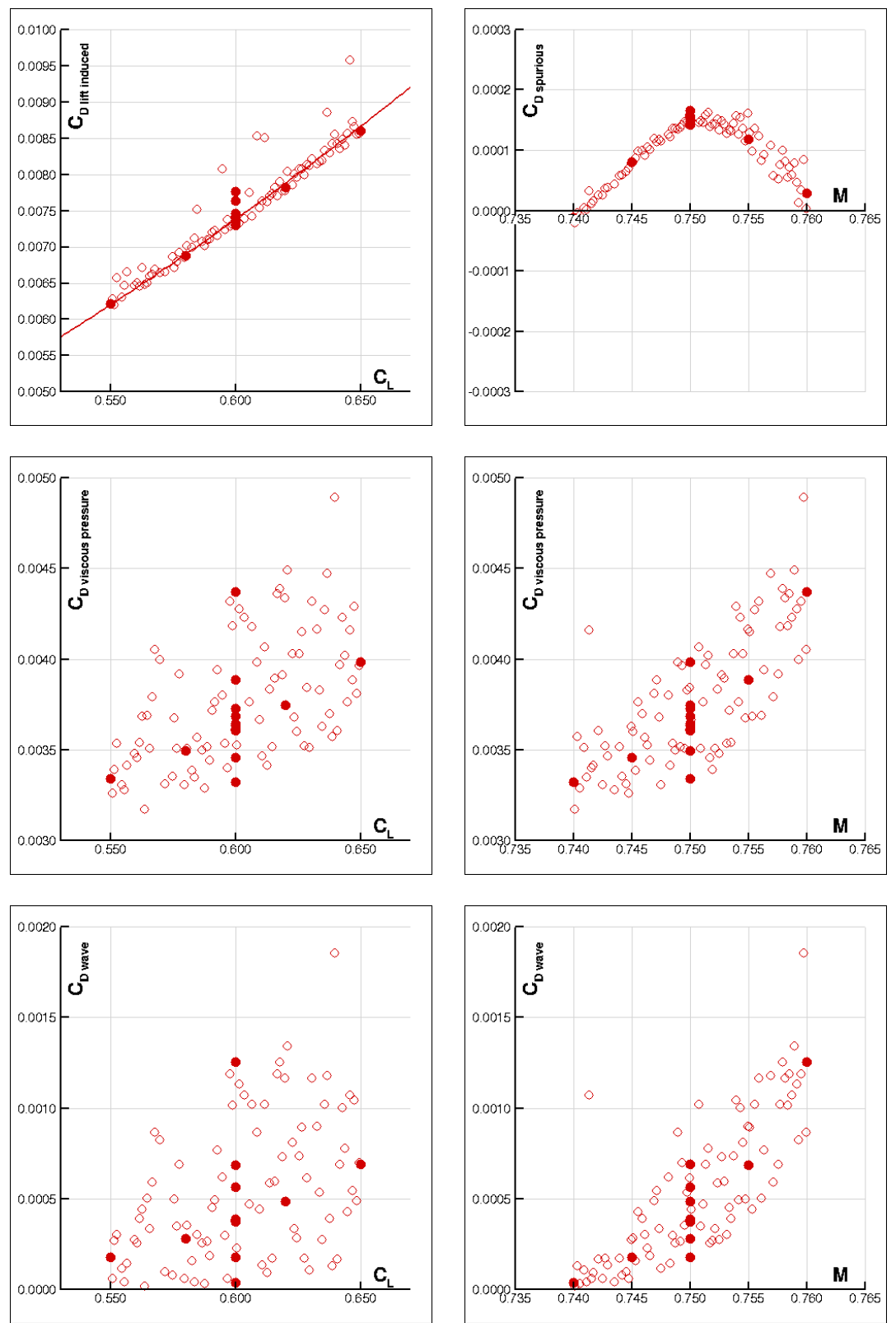

Figure 12: Noticeable input-output dependencies for aerodynamics $(\bullet$ manual sampling set, ○ LHS set). 

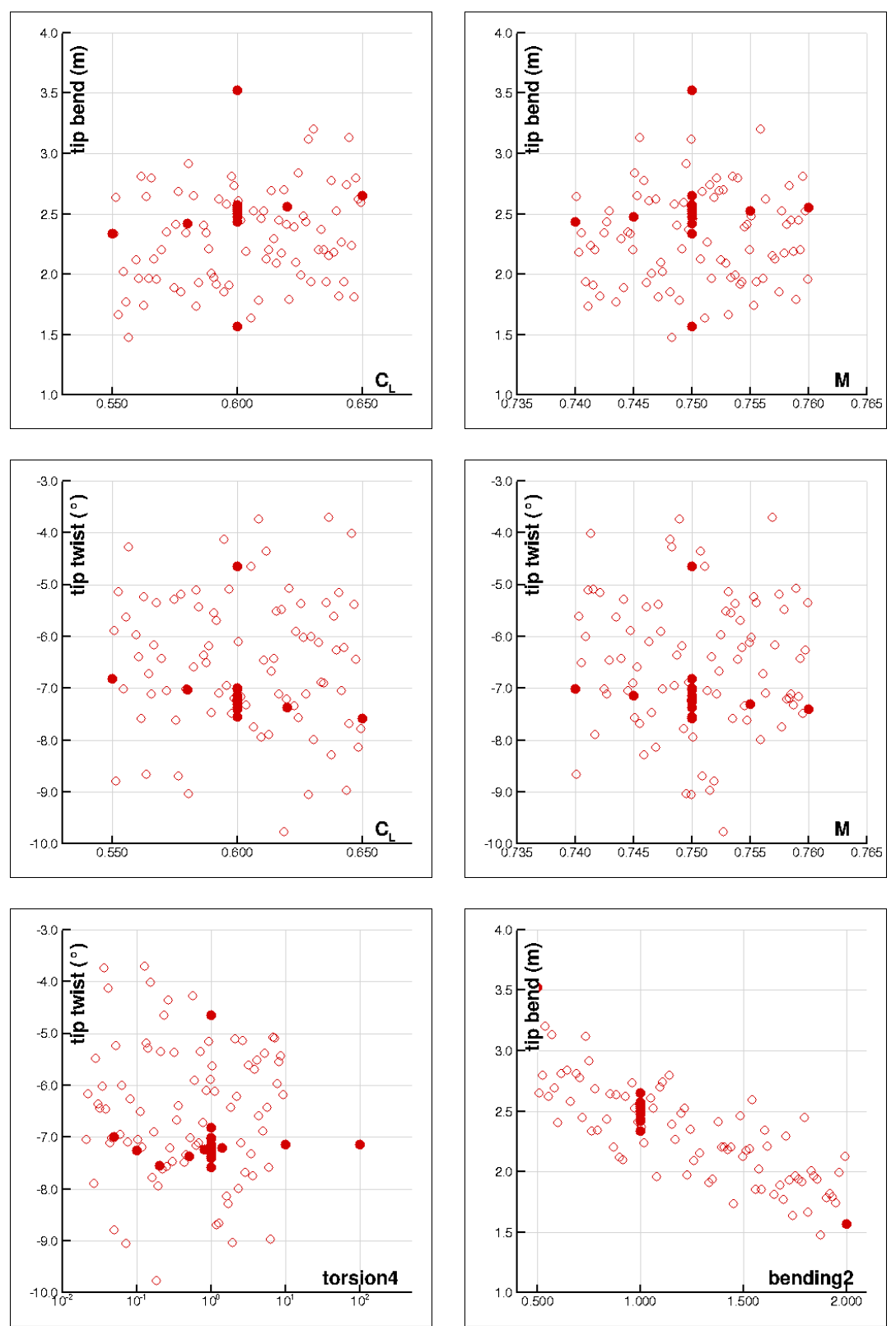

Figure 13: Noticeable input-output dependencies for aeroelasticity $(\bullet$ manual sampling set, ○ LHS set). 

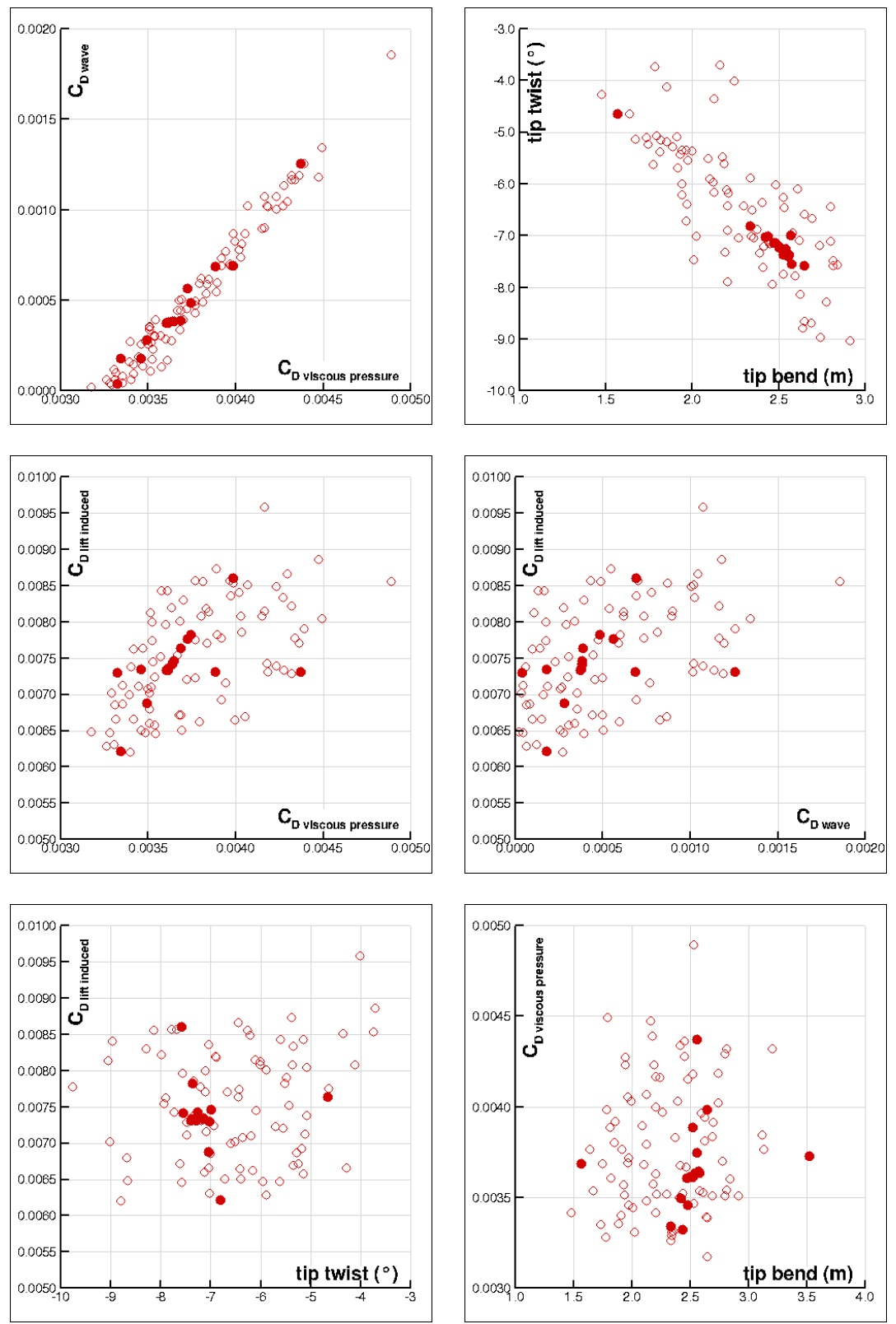

Figure 14: Noticeable output-output dependencies (• manual sampling set, $\circ$ LHS set). 


\subsection{Polynomial surrogates}

Polynomial surrogates for aerodynamic computations have been outlined in e.g. [49]. We basically follow this presentation here. Let $g$ be a generic physical/numerical model involving $D$ parameters $\boldsymbol{\xi}=\left(\xi_{1}, \xi_{2}, \ldots \xi_{D}\right) \in \mathcal{I} \subseteq$ $\mathbb{R}^{D}$, such that the quantity of interest $y \in \mathcal{Y}$ is given as:

$$
y=g(\boldsymbol{\xi}) .
$$

Let $\mathbb{P}^{q}[\boldsymbol{x}]$ be the set of $D$-dimensional polynomials with total order $q$ with respect to $\boldsymbol{x} \in \mathbb{R}^{D}$. We first note that this set has cardinality $\# \mathbb{P}^{q}[\boldsymbol{x}]=P+1$ such that:

$$
P+1=\frac{(q+D) !}{q ! D !} .
$$

A polynomial surrogate model $\hat{g}_{q}$ of order $q$ for the model $g$ is obtained as:

$$
g \approx \hat{g}_{q}=\arg \min _{\pi \in \mathbb{P}^{q}[\boldsymbol{x}]} \frac{1}{2} \int_{\mathbb{R}^{D}}|g(\boldsymbol{x})-\pi(\boldsymbol{x})|^{2} \mathcal{P}_{\boldsymbol{\Xi}}(\mathrm{d} \boldsymbol{x})
$$

where $\boldsymbol{\Xi} \sim \mathcal{P}_{\boldsymbol{\Xi}}$ is the marginal PDF of the random parameters $\boldsymbol{\Xi}$ with values in $\mathcal{I} \subseteq \mathbb{R}^{D}$. The accuracy of this approximation may be assessed considering the limit of the mean-square norm $\mathbb{E}\left\{\left|\hat{g}_{q}(\boldsymbol{\Xi})-g(\boldsymbol{\Xi})\right|^{2}\right\}$ as $q \rightarrow+\infty$. However such a "convergence" does not necessarily holds, and it depends on the probability measure $\mathcal{P}_{\Xi}$.

There are several ways to construct polynomial response surfaces and surrogates: embedded projection (this is the original spectral stochastic finite element method of Sun [56] and Ghanem \& Spanos [25] which is highly intrusive), non-intrusive projection (e.g. collocation) [28, 64], or regression, whereby an $\ell_{2}$-optimization problem is formed; see also Le Maître \& Knio [37]

for a detailed introduction. For that purpose, a set of sampling points $\left\{\boldsymbol{\xi}_{\ell}\right\}_{\ell=1}^{N}$ 
where the model $g$ has been evaluated, is needed in order to discretize the minimization problem (12). These observations are denoted by $\left\{y_{\ell}\right\}_{\ell=1}^{N}$ where $y_{\ell}=g\left(\boldsymbol{\xi}_{\ell}\right), 1 \leq \ell \leq N$.

\subsubsection{Polynomial chaos expansion}

From now on we assume that an orthonormal polynomial basis $\mathcal{B}$ of $L^{2}\left(\mathcal{I}, \mathcal{P}_{\Xi}\right)$ is available. Then we construct the polynomial surrogate $\hat{g}_{q}$ of $g$ by standard $L^{2}$ projection on the finite dimensional subspace of $L^{2}\left(\mathcal{I}, \mathcal{P}_{\Xi}\right)$ spanned by the truncated family of orthonormal polynomials up to the total order $q$ denoted by $\mathcal{B}^{q}=\left\{\psi_{j}\right\}_{j=0}^{P}$, where $P$ is again given by Eq. (11). The orthonormalization of this basis reads:

$$
\int_{\mathcal{I}} \psi_{j}(\boldsymbol{x}) \psi_{k}(\boldsymbol{x}) \mathcal{P}_{\Xi}(\mathrm{d} \boldsymbol{x})=\left(\psi_{j}, \psi_{k}\right)_{L^{2}}=\delta_{j k} ;
$$

then:

$$
\hat{g}_{q}(\boldsymbol{x})=\sum_{j=0}^{P} g_{j} \psi_{j}(\boldsymbol{x})=\sum_{j=0}^{P}\left(g, \psi_{j}\right)_{L^{2}} \psi_{j}(\boldsymbol{x}) .
$$

Such representations are referred to as polynomial chaos (PC) expansions in the dedicated literature, provided that the variable parameters $\boldsymbol{\Xi}$ follow a multi-dimensional Gaussian (normal) distribution $\mathcal{P}_{\Xi}=\bigotimes_{d=1}^{D} \mathcal{N}(0,1)$ $[25,37]$. They are otherwise called generalized polynomial chaos (gPC) expansions for other distributions $[\underline{53}, \underline{63}]$.

The sampling points are typically used to evaluate the expansion coefficients $g_{j}=\left(g, \psi_{j}\right)_{L^{2}}$ in the foregoing representation. For example, they can be chosen as the $N$ integration points of a quadrature rule $\boldsymbol{\Theta}^{N}=\left\{\omega_{\ell}, \boldsymbol{\xi}_{\ell}\right\}_{\ell=1}^{N}$, which provides with positive weights $\left\{\omega_{\ell}\right\}_{\ell=1}^{N}$ and nodes $\left\{\boldsymbol{\xi}_{\ell}\right\}_{\ell=1}^{N}$ in $\mathbb{R}^{D}$ such 
that for a smooth function $\boldsymbol{x} \mapsto f(\boldsymbol{x})$ one can evaluate its average by:

$$
\int_{\mathcal{I}} f(\boldsymbol{x}) \mathcal{P}_{\boldsymbol{\Xi}}(\mathrm{d} \boldsymbol{x}) \simeq \sum_{\ell=1}^{N} \omega_{\ell} f\left(\boldsymbol{\xi}_{\ell}\right)
$$

Using the quadrature rule above, the expansion coefficients are approximated by:

$$
g_{j} \approx g_{j}^{N}=\sum_{\ell=1}^{N} \omega_{\ell} y_{\ell} \psi_{j}\left(\boldsymbol{\xi}_{\ell}\right), \quad 0 \leq j \leq P .
$$

This corresponds to the approximation $\hat{g}_{q} \approx \hat{g}_{q}^{N}=\sum_{j=0}^{P} g_{j}^{N} \psi_{j}$. Classical Gauss-Jacobi quadratures require $q$ nodes to exactly integrate one-dimensional polynomials of order $2 q-1$. The polynomial basis $\mathcal{B}^{q}$ adapted to the parameters $\operatorname{PDF} \mathcal{P}_{\Xi}$ is constituted by the $D$-dimensional orthonormal polynomials $\psi_{\boldsymbol{j}}, \boldsymbol{j}=\left(j_{1}, j_{2}, \ldots j_{D}\right) \in \mathbb{N}^{D}$, such that $\|\boldsymbol{j}\|_{1}=\sum_{d=1}^{D} j_{d} \leq q$ (note that they are $P+1$ such multi-indices according to Eq. (11) and it is understood here and below that they are simply reordered as single indices in the PC expansion (14)). These polynomials are given by:

$$
\psi_{\boldsymbol{j}}(\boldsymbol{x})=\prod_{d=1}^{D} \psi_{j_{d}}\left(x_{d}\right), \quad\|\boldsymbol{j}\|_{1} \leq q,
$$

where $\left\{\psi_{j_{d}}\right\}_{j_{d} \geq 0}$ is the family of one-dimensional orthonormal polynomials with respect to the PDF of the $d$-th variable parameter. Therefore, $N \approx\left\lfloor\frac{q}{2}\right\rfloor^{D}$ sampling points are needed to exactly integrate $D$-dimensional polynomials of total order $q$. Using sparse quadrature rules $[23, \underline{44}, \underline{52}]$ yields $N \approx 2^{\left\lfloor\frac{q}{2}\right\rfloor} P$ whenever $D \gg 1$, which may still be unaffordable for a complex model $g$ : this is the so-called curse of dimensionality. Practical examples, though, reveal that the vector $\boldsymbol{g}=\left(g_{0}, g_{1}, \ldots g_{P}\right)^{\top} \in \mathbb{R}^{P+1}$ of the expansion coefficients of $\hat{g}_{q}$ can have many negligible components [49], so that it is compressible in the 
terminology of the theory of compressed sensing, or compressive sampling (CS) $[\underline{8}-10,19]$. In other words, its sparsity:

$$
S=\#\left\{j ;\left|g_{j}\right|>\delta\right\}
$$

for some tolerance $0 \leq \delta \ll \mathbb{E}\left\{\left|\hat{g}_{q}(\boldsymbol{\Xi})\right|\right\}$, say, is such that $S \ll P$. In this setting it is argued that only a number of samples $N$ proportional to the compressed size $S$, rather than the uncompressed size $P$, of the sought signal (in the present case the surrogate model $\hat{g}_{q}$ ) is needed in order to reconstruct it. The reconstruction of the expansion coefficients from a sparsity argument is outlined in the next section. There we basically follow the introductory paper of Candès \& Wakin [10] on the underlying CS theory.

\subsubsection{Non-adapted sparse reconstruction by $\ell_{1}-$ minimization}

Evaluating the gPC expansion (14) at the sampling points $\boldsymbol{\Theta}^{N}=\left\{\boldsymbol{\xi}_{\ell}\right\}_{\ell=1}^{N}$ we arrive at the $N \times(P+1)$ system:

$$
\boldsymbol{y}=\boldsymbol{M g}+\boldsymbol{\epsilon}
$$

where $\boldsymbol{y}=\left(y_{1}, y_{2}, \ldots y_{N}\right)^{\top} \in \mathcal{Y}^{N}$ is the vector of observations, and $\boldsymbol{M}$ is the so-called $N \times(P+1)$ measurement matrix given by $[\boldsymbol{M}]_{\ell j}=\psi_{j}\left(\boldsymbol{\xi}_{\ell}\right)$. Since the gPC expansion truncated at the total order $q$ is not complete for the exact representation of $g$, a truncation error vector $\boldsymbol{\epsilon}$ has been accounted for in the foregoing process. Eq. $\underline{(17)}$ is ill-posed and has infinitely many solutions whenever $N \ll P$, unless additional constraints of the sought solution $\boldsymbol{g}$ are accounted for. Regularized versions of Eq. (17) exist for this case, which in turn ensure its well-posedness. Together with a sparsity constraint, this can be accommodated by reformulating Eq. $(17)$ as the following $\ell_{0}-$ minimization 
problem where a sparsest approximation is sought for:

$$
\boldsymbol{g} \approx \boldsymbol{g}^{\star}=\arg \min _{\boldsymbol{h} \in \mathbb{R}^{P+1}}\left\{\|\boldsymbol{h}\|_{0} ;\|\boldsymbol{M h}-\boldsymbol{y}\|_{2} \leq \varepsilon\right\},
$$

for some tolerance $0 \leq \varepsilon \ll 1$ on the polynomial chaos truncation; the norms above are defined by $\|\boldsymbol{h}\|_{m}=\left(\sum_{j=0}^{P}\left|h_{j}\right|^{m}\right)^{\frac{1}{m}}$ for $m>0$ and $\|\boldsymbol{h}\|_{0}=$ $\#\left\{j ; h_{j} \neq 0\right\}$ otherwise. The solution of the problem $\left(P_{0,0}\right)$ is not always unique and the latter is also NP-hard to solve, that is, the time needed to solve it is exponential with respect to $P$ (as for example the brute force algorithm for optimization). However it can be relaxed by convex $\ell_{1}-$ minimization, known as Basis Pursuit Denoising (BPDN) [13]:

$$
\boldsymbol{g} \approx \boldsymbol{g}^{\star}=\arg \min _{\boldsymbol{h} \in \mathbb{R}^{P+1}}\left\{\|\boldsymbol{h}\|_{1} ;\|\boldsymbol{M} \boldsymbol{h}-\boldsymbol{y}\|_{2} \leq \varepsilon\right\} .
$$

For sufficiently sparse coefficients $\boldsymbol{g}$ and some conditions on the measurement matrix $\boldsymbol{M},\left(P_{0,0}\right)$ and $\left(P_{1,0}\right)$ share the same unique solution. Consequently the strategy for the present study is to solve $\underline{\left(P_{1, \varepsilon}\right)}$ with $N$ runs of the aeroelastic model $g$ significantly lower than the number of coefficients to be identified. Indeed CS theory shows that it is relevant provided that the target solution $\boldsymbol{g}$ is actually sparse, or nearly sparse (compressive), and some constraints on the measurement matrix are fulfilled.

One of such requirements for the successful recovery of a sparse vector by $\underline{\left(P_{1, \varepsilon}\right)}$ is small mutual coherence of the columns of the measurement matrix, or their near orthogonality. The coherence $0<\mu\left(\boldsymbol{\Theta}^{N}, \mathcal{B}^{q}\right) \leq 1$ :

$$
\mu\left(\boldsymbol{\Theta}^{N}, \mathcal{B}^{q}\right)=\max _{\substack{1 \leq j, k \leq P+1 \\ j \neq k}} \frac{\left|\boldsymbol{m}_{j}^{\top} \boldsymbol{m}_{k}\right|}{\left\|\boldsymbol{m}_{j}\right\|_{2}\left\|\boldsymbol{m}_{k}\right\|_{2}},
$$

where $\boldsymbol{m}_{j}=\left(\psi_{j}\left(\boldsymbol{\xi}_{1}\right), \psi_{j}\left(\boldsymbol{\xi}_{2}\right), \ldots \psi_{j}\left(\boldsymbol{\xi}_{N}\right)\right)^{\mathrm{T}}$ stands for the $j$-th column of $\boldsymbol{M}$, is a measure of how close to orthogonality the measurement matrix is. Based on 
this coherency measure, the following theorem from Candès \& Romberg [9] asserts that if $\hat{g}_{q}$ is sufficiently sparse in $\mathcal{B}^{q}$, the recovery of its gPC coefficients by $\ell_{1}-$ minimization is exact.

Theorem 3.1. Assume that $\hat{g}_{q}$ is $S$-sparse on the $g P C$ basis $\mathcal{B}^{q}$, that is $\boldsymbol{g}$ has at most $S$ non-zero entries. Then if $N$ sampling points $\boldsymbol{\Theta}^{N}=\left\{\boldsymbol{\xi}_{\ell}\right\}_{\ell=1}^{N}$ are selected at random to form the measurement matrix $\boldsymbol{M}$, and:

$$
N \geq C \cdot \mu\left(\Theta^{N}, \mathcal{B}^{q}\right) \cdot S \cdot \log P
$$

for some constant $C>0$, the solution of $\left(P_{1,0}\right)$ is exact with "overwhelming" (sic) probability.

More precise results with structured random measurement matrices are given by e.g. Rauhut \& Ward [48]. It should be noted that the role of coherence in this result is transparent. The smaller the coherence is, the closer the measurement matrix is to a unitary matrix, and the fewer sampling points are needed. This result also states that $\left(P_{1,0}\right)$ can be effectively solved without any prior knowledge of the number of non-zero coefficients, their orders (locations), or their amplitudes. In other words, the reconstruction procedure permitted by the CS theory is non adapted because it identifies the sparsity pattern of a signal in its sparsifying basis, and the leading components at the same time. This procedure can therefore efficiently capture the relevant information of a sparse signal without trying to comprehend that signal [10] contrary to the proposal of [ $\underline{5}$. This is clearly a much desirable feature for practical industrial applications.

The previous Theorem 3.1 is however not entirely satisfactory from a practical point of view because (i) it does not allow for some truncation er- 
ror, or noisy/imprecise observations; (ii) it does not deal with approximately sparse vectors, for which a large subset of entries are negligible rather than strictly zero. These shortcomings may be alleviated simultaneously as established by Candès et al. [8]. To achieve this, a constraint on the measurement matrix $\boldsymbol{M}$ needs be added to gain robustness in CS, the so-called restricted isometry property (RIP) also quoted as the uniform uncertainty principle. For each integer $S \in \mathbb{N}^{*}$, the isometry constant $\delta_{S}$ of $\boldsymbol{M}$ is defined as the smallest number such that:

$$
\left(1-\delta_{S}\right)\left\|\boldsymbol{h}_{S}\right\|_{2}^{2} \leq\left\|\boldsymbol{M} \boldsymbol{h}_{S}\right\|_{2}^{2} \leq\left(1+\delta_{S}\right)\left\|\boldsymbol{h}_{S}\right\|_{2}^{2}
$$

for all $S$-sparse vectors $\boldsymbol{h}_{S} \in \mathcal{Y}_{S}:=\left\{\boldsymbol{h} \in \mathbb{R}^{P+1} ;\|\boldsymbol{h}\|_{0} \leq S\right\}$. Then $\boldsymbol{M}$ is said to satisfy the RIP of order $S$ if, say, $\delta_{S}$ is not too close to 1 . This property amounts to saying that all $S$-column submatrices of $\boldsymbol{M}$ are numerically well-conditioned, or $S$ columns $\boldsymbol{m}_{j_{1}}, \boldsymbol{m}_{j_{2}} \ldots \boldsymbol{m}_{j_{S}}$ selected arbitrarily in $\boldsymbol{M}$ are nearly orthogonal. The following theorem by Candès et al. $[\underline{8}, \underline{10}]$ then states that $\underline{\left(P_{1, \varepsilon}\right)}$ can be solved efficiently:

Theorem 3.2. Assume $\delta_{2 S}<\sqrt{2}-1$. Then the solution $\boldsymbol{g}^{\star}$ to $\underline{\left(P_{1, \varepsilon}\right)}$ satisfies:

$$
\left\|\boldsymbol{g}^{\star}-\boldsymbol{g}\right\|_{2} \leq C_{0} \frac{\left\|\boldsymbol{g}_{S}-\boldsymbol{g}\right\|_{1}}{\sqrt{S}}+C_{1} \varepsilon
$$

for some $C_{0}, C_{1}>0$. Here $\boldsymbol{g}_{S}$ is $\boldsymbol{g}$ with all but the $S$ largest entries set to zero.

This result calls for several comments. First, it is more general than Theorem 3.1 since, if the signal is exactly $S$-sparse, $\boldsymbol{g}=\boldsymbol{g}_{S}$ and the reconstruction is exact whenever $\varepsilon=0$ (noiseless case). Second, it deals with all signals, not 
the $S$-sparse ones solely. Third, it is deterministic and does not involve any probability. Lastly, the bound $\sqrt{2}-1$ on $\delta_{2 S}$ is the one originally proposed by Candès \& Wakin [10] but it can be improved as proposed by e.g. Mo \& Li [42]; such improvements are an active field of research at present.

\subsubsection{Application to Uncertainty Quantification (UQ)}

Once the polynomial surrogate model $\hat{g}_{q}$ has been derived, a mean output functional of the quantity of interest $y$ can be estimated by:

$$
\mathbb{E}\{f(y)\} \simeq \int_{\mathcal{I}} f\left(\hat{g}_{q}(\boldsymbol{x})\right) \mathcal{P}_{\boldsymbol{\Xi}}(\mathrm{d} \boldsymbol{x})
$$

where $y \mapsto f(y)$ is a regular function on $\mathcal{Y}$. The mean $\mu$ is obtained for $f(y)=y$, the variance $\sigma^{2}$ is obtained for $f(y)=(y-\mu)^{2}$, the skewness $\gamma_{1}$ for $f(y)=\left(\frac{y-\mu}{\sigma}\right)^{3}$, the kurtosis $\beta_{2}$ for $f(y)=\left(\frac{y-\mu}{\sigma}\right)^{4}$, etc. Owing to the orthonormality $\left(\underline{13)}\right.$ of the polynomials of $\mathcal{B}^{q}$ the mean is simply $\mu=g_{0}$ and the variance is $\sigma^{2}=\sum_{j=1}^{P} g_{j}^{2}$. Higher-order moments may be computed with the formulas derived in [50] for the continuous orthogonal polynomials of the Askey scheme.

Sensitivity indices may be computed alike. Denoting by $\mathscr{I}_{d}$ the set of indices corresponding to the polynomials of $\mathcal{B}^{q}$ depending only on the $d$-th variable parameter $\xi_{d}$, the main-effect gPC-based Sobol' indices are given by (see for example [55]):

$$
\mathscr{S}_{d}=\frac{1}{\sigma^{2}} \sum_{j \in \mathscr{I}_{d}} g_{j}^{2},
$$

invoking again the normalization condition $\underline{(13)}$. More generally, if $\mathscr{I}_{d_{1} d_{2} \ldots d_{s}}$ is the set of indices corresponding to the polynomials of $\mathcal{B}^{q}$ depending only 
on the parameters $\xi_{d_{1}}, \xi_{d_{2}}, \ldots \xi_{d_{s}}$, the $s$-fold joint sensitivity indices are:

$$
\mathscr{S}_{d_{1} d_{2} \ldots d_{s}}=\frac{1}{\sigma^{2}} \sum_{j \in \mathscr{I}_{d_{1} d_{2} \ldots d_{s}}} g_{j}^{2} .
$$

In the following application to the aeroelastic database, we will primarily consider the main-effect sensitivity indices $\mathscr{S}_{d}$.

\subsubsection{Application to the aeroelastic database}

We now apply the foregoing procedure to the non-adaptive computation of the gPC coefficients $\boldsymbol{g}$ of the surrogate models $\hat{g}_{q}$ for the output quantities of interest of Section 2.3.3. We use the $N=102$ sampling points drawn manually and by LHS as explained in Section 2.3, so that the chaos basis $\mathcal{B}^{q}$ is constituted by Legendre polynomials because the latter are orthogonal with respect to the uniform measure. Note in passing that the uniform probability measure is the one arising from Jaynes' maximum entropy principle $[\underline{33}, \underline{34}]$ when the sole imposed constraint is the compactness of its support. We also note that techniques have been developed to enrich an LHS database with inherited samples in e.g. [60]. We consider a total order $q=3$, thus $P=285$ since $D=10$ here, with $\xi_{1}=M, \xi_{2}=C_{L}, \xi_{3}$ through $\xi_{6}$ for the bending parameters, and $\xi_{7}$ through $\xi_{10}$ for the torsion parameters. The coherence for the present sampling set and representation basis is $\mu\left(\Theta^{102}, \mathcal{B}^{3}\right) \simeq 0.94$ and the sparsity of the polynomial surrogates is observed to be $S \simeq 10$. Thus Eq. (19) yields $N \gtrsim 50$. A common observation is that $N \gtrsim 4 S \simeq 40$ is usually enough for a successful recovery.

We subsequently apply $\operatorname{BPDN} \underline{\left(P_{1, \varepsilon}\right)}$ to compute $\boldsymbol{g}$. For that purpose we use the Spectral Projected Gradient Algorithm (SPGL1) developed by van den Berg \& Friedlander [58] and implemented in the Matlab package 
SPGL1 [57] to solve this $\ell_{1}-$ minimization problem. The tolerance was fixed at $\varepsilon=10^{-5}$ and we were able to find a solution for all surrogates with this a priori choice without resorting to cross-validation, for example. Further investigations should be carried on on this topic, though. It should also be noted that no particular sampling strategy, such as stratification, lowdiscrepancy series, or preconditioning, has been applied at this stage to select the sampling set. Alternative strategies are outlined in several recent works; see for example $[20, \underline{29}, \underline{40}, \underline{45}, \underline{48}, \underline{51}]$.

The root mean-square errors of the output quantities of interest $C_{m}$ (pitching moment coefficient), $C_{D s}=C_{D \text {,pressure }}+C_{D \text {,friction }}$ (skin drag coefficient), $C_{D v}=C_{D \text {,lift induced }}+C_{D \text {, wave }}+C_{D \text {,viscous pressure }}+C_{D \text {,friction }}$ (far-field drag coefficient), $\alpha$ (angle of attack), $U$ (wing tip bend), and $\varphi$ (wing tip twist) are gathered in the Table $\underline{2}$. The root mean-square error $e_{2}$ is defined by (note that it is directly related to the chosen tolerance $\varepsilon$ ):

$$
e_{2}=\sqrt{\frac{\sum_{\ell=1}^{N}\left|y_{\ell}-\hat{g}_{q}\left(\boldsymbol{\xi}_{\ell}\right)\right|^{2}}{\sum_{\ell=1}^{N}\left|y_{\ell}\right|^{2}}} .
$$

The mean $\mu$ and standard deviation $\sigma$ are gathered in the Table $\underline{2}$ as well, while the main-effect sensitivity indices $\mathscr{S}_{1}$ through $\mathscr{S}_{10}$ of Eq. (20) are gathered in Table 3 . The strong dependence of the wing tip bend $U$ on the second bending parameter $\xi_{4}$ underlined in Section 2.3.4, for example, is recovered from these results $\left(\mathscr{S}_{4}=62 \%\right)$. The noticeable dependence of the wing tip twist $\varphi$ on the third bending parameter $\xi_{5}$ may also be observed $\left(\mathscr{S}_{5}=32 \%\right)$. The PDFs are displayed on Fig. 15 . They were estimated from $N_{s}=100,000$ 
interrogations of the gPC surrogates $\hat{g}_{q}$ and smoothing out the resulting histograms by a normal kernel density function [59]. They are compared with a standard Gaussian distribution with the same mean and standard deviation. These comparisons are quantified by the Kullback-Leibler divergence $D_{\mathrm{KL}}(\mathcal{P} \| \mathcal{Q})$ defined by $[35]$ :

$$
D_{\mathrm{KL}}(\mathcal{P} \| \mathcal{Q})=\int_{\mathcal{Y}} \log \frac{\mathcal{P}(y)}{\mathcal{Q}(y)} \mathcal{P}(\mathrm{d} y),
$$

where $\mathcal{P}$ is the reconstructed marginal PDF of the output quantity of interest $y$, and $\mathcal{Q} \equiv \mathcal{N}(\mu, \sigma)$ is the standard Gaussian distribution it is compared to. It is computed in the Table $\underline{2}$ below for the various output quantities of interest considered here.

\begin{tabular}{|c|c|c|c|}
\hline & $C_{m}$ & $C_{D s}$ & $C_{D v}$ \\
\hline$\mu$ & $-11.63 \mathrm{e}-02$ & $219.83 \mathrm{e}-04$ & $218.69 \mathrm{e}-04$ \\
\hline$\sigma$ & $1.55 \mathrm{e}-02$ & $6.53 \mathrm{e}-04$ & $6.28 \mathrm{e}-04$ \\
\hline$e_{2}$ & $4.70 \mathrm{e}-03$ & $0.45 \mathrm{e}-03$ & $0.35 \mathrm{e}-03$ \\
\hline$D_{\mathrm{KL}}(\mathcal{P} \| \mathcal{Q})$ & $1.10 \mathrm{e}-02$ & $1.11 \mathrm{e}-02$ & $1.39 \mathrm{e}-02$ \\
\hline & $\alpha\left(^{\mathrm{O}}\right)$ & $U(\mathrm{~m})$ & $\varphi\left(^{\mathrm{o}}\right)$ \\
\hline$\mu$ & 2.53 & 2.16 & -6.10 \\
\hline$\sigma$ & 0.20 & 0.26 & 0.80 \\
\hline$e_{2}$ & $1.88 \mathrm{e}-03$ & $1.65 \mathrm{e}-03$ & $3.97 \mathrm{e}-03$ \\
\hline$D_{\mathrm{KL}}(\mathcal{P} \| \mathcal{Q})$ & $1.40 \mathrm{e}-02$ & $0.50 \mathrm{e}-02$ & $0.62 \mathrm{e}-02$ \\
\hline
\end{tabular}

Table 2: Root mean-square error, mean, standard deviation, and Kullback-Leibler divergence from a Gaussian distribution of the output quantities of interest computed by $\ell_{1}-$ minimization with $N=102$ sampling points. 


\begin{tabular}{|c||c|c|c|}
\hline & $C_{m}$ & $C_{D s}$ & $C_{D v}$ \\
\hline \hline $\mathscr{S}_{1}$ & $0.048 \mathrm{e}-01$ & $2.657 \mathrm{e}-01$ & $2.286 \mathrm{e}-01$ \\
$\mathscr{S}_{2}$ & $0.036 \mathrm{e}-01$ & $1.841 \mathrm{e}-01$ & $1.682 \mathrm{e}-01$ \\
$\mathscr{S}_{3}$ & $0.052 \mathrm{e}-01$ & $0.136 \mathrm{e}-01$ & $0.136 \mathrm{e}-01$ \\
$\mathscr{S}_{4}$ & $0.229 \mathrm{e}-01$ & $0.000 \mathrm{e}-01$ & $0.002 \mathrm{e}-01$ \\
$\mathscr{S}_{5}$ & $0.046 \mathrm{e}-01$ & $0.000 \mathrm{e}-01$ & $0.002 \mathrm{e}-01$ \\
$\mathscr{S}_{6}$ & $0.280 \mathrm{e}-01$ & $0.038 \mathrm{e}-01$ & $0.027 \mathrm{e}-01$ \\
$\mathscr{S}_{7}$ & $0.078 \mathrm{e}-01$ & $0.002 \mathrm{e}-01$ & $0.004 \mathrm{e}-01$ \\
$\mathscr{S}_{8}$ & $0.005 \mathrm{e}-01$ & $0.001 \mathrm{e}-01$ & $0.001 \mathrm{e}-01$ \\
$\mathscr{S}_{9}$ & $0.322 \mathrm{e}-01$ & $0.115 \mathrm{e}-01$ & $0.149 \mathrm{e}-01$ \\
$\mathscr{S}_{10}$ & $0.285 \mathrm{e}-01$ & $0.073 \mathrm{e}-01$ & $0.191 \mathrm{e}-01$ \\
\hline
\end{tabular}

\begin{tabular}{|c||c|c|c|}
\hline & $\alpha$ & $U$ & $\varphi$ \\
\hline \hline $\mathscr{S}_{1}$ & $0.003 \mathrm{e}-01$ & $0.002 \mathrm{e}-01$ & $0.105 \mathrm{e}-01$ \\
$\mathscr{S}_{2}$ & $1.333 \mathrm{e}-01$ & $0.007 \mathrm{e}-01$ & $0.011 \mathrm{e}-01$ \\
$\mathscr{S}_{3}$ & $0.005 \mathrm{e}-01$ & $0.974 \mathrm{e}-01$ & $0.021 \mathrm{e}-01$ \\
$\mathscr{S}_{4}$ & $0.154 \mathrm{e}-01$ & $6.187 \mathrm{e}-01$ & $0.461 \mathrm{e}-01$ \\
$\mathscr{S}_{5}$ & $0.001 \mathrm{e}-01$ & $1.137 \mathrm{e}-01$ & $3.186 \mathrm{e}-01$ \\
$\mathscr{S}_{6}$ & $0.010 \mathrm{e}-01$ & $0.016 \mathrm{e}-01$ & $0.128 \mathrm{e}-01$ \\
$\mathscr{S}_{7}$ & $0.840 \mathrm{e}-01$ & $0.196 \mathrm{e}-01$ & $0.774 \mathrm{e}-01$ \\
$\mathscr{S}_{8}$ & $0.722 \mathrm{e}-01$ & $0.109 \mathrm{e}-01$ & $0.506 \mathrm{e}-01$ \\
$\mathscr{S}_{9}$ & $0.003 \mathrm{e}-01$ & $0.010 \mathrm{e}-01$ & $0.034 \mathrm{e}-01$ \\
$\mathscr{S}_{10}$ & $0.390 \mathrm{e}-01$ & $0.057 \mathrm{e}-01$ & $0.411 \mathrm{e}-01$ \\
\hline
\end{tabular}

Table 3: Main-effect sensitivity indices of the output quantities of interest computed by $\ell_{1}-$ minimization with $N=102$ sampling points. 

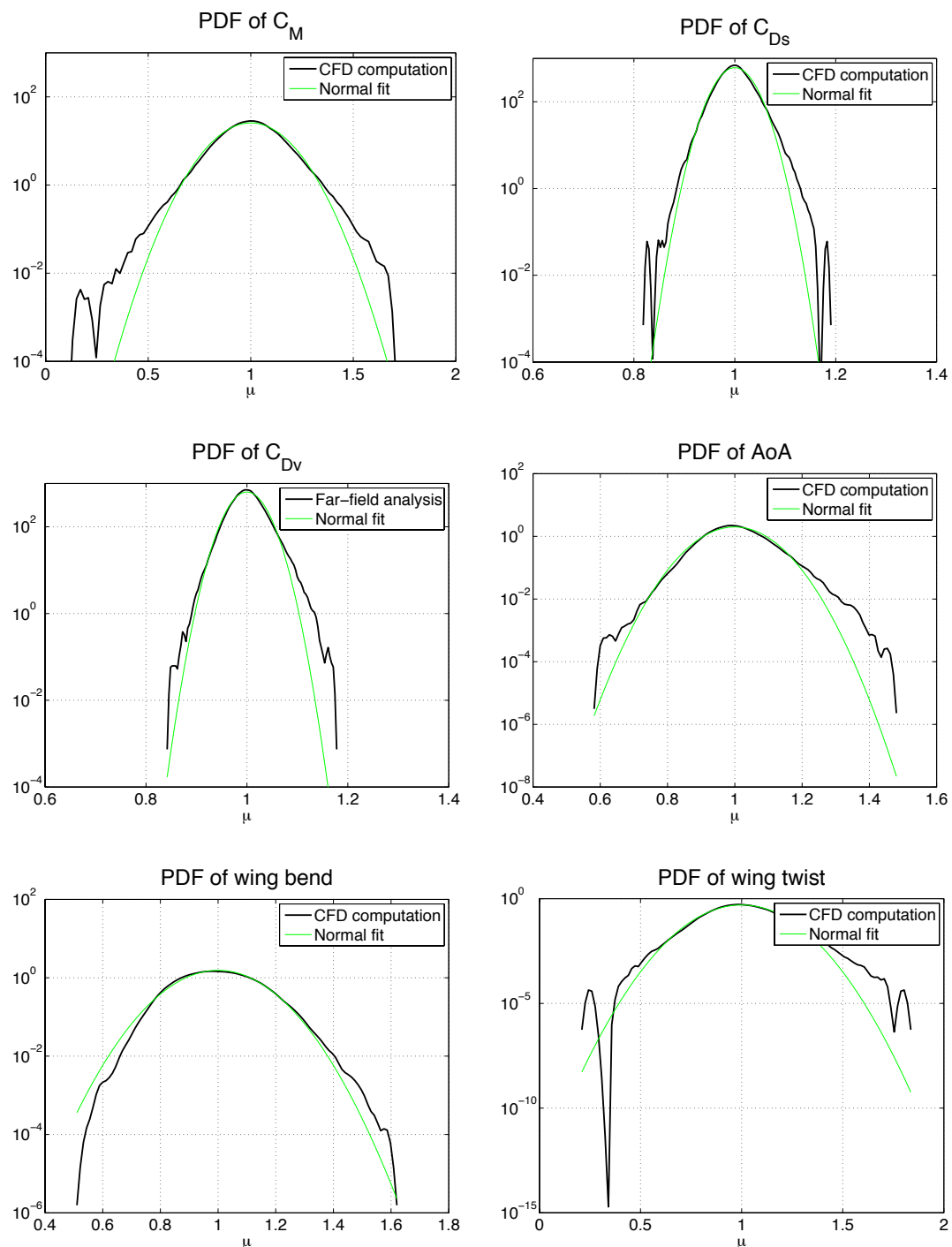

Figure 15: PDFs of the pitching moment coefficient (top left), skin drag coefficient (top right), far-field drag coefficient (middle left), angle of attack (middle right), wing tip bend (bottom left), and wing tip twist (bottom right) computed by $\ell_{1}$-minimization with $N=102$ sampling points. Comparison with a standard Gaussian distribution (green curve) with the same mean and standard deviation. 
In view of the comments of Section 2.3.4, we have also quantified the dependence of the lift-induced drag coefficient $C_{D \text {,lift induced and spurious drag }}$ coefficient $C_{D \text {,spurious }}=C_{D s}-C_{D v}$ on the various parameters. The tolerance for the computation of their $\mathrm{gPC}$ coefficients by $\underline{\left(P_{1, \varepsilon}\right)}$ was fixed at $\varepsilon=10^{-6}$ and $\varepsilon=10^{-9}$, respectively. The root mean-square error, mean, standard deviation, and Kullback-Leibler divergence from a Gaussian distribution are gathered in the Table $\underline{4}$, while the main-effect sensitivity indices are gathered in Table $\underline{5}$. Their PDFs are displayed on Fig. 16, using again $N_{s}=100,000$ evaluations of the gPC surrogates and smoothing with a normal kernel density function. These results confirm the strong dependence of $C_{D, \text { spurious }}$ on the Mach number $\xi_{1}$ (for $\mathscr{S}_{1}=94 \%$ ), and to a lesser extent that of $C_{D, \text { lift induced }}$ on the lift coefficient $\xi_{2}$ (for $\mathscr{S}_{2}=48 \%$ ).

\begin{tabular}{|c||c|c|}
\hline & $C_{D, \text { lift induced }}$ & $C_{D, \text { spurious }}$ \\
\hline \hline$\mu$ & $72.60 \mathrm{e}-04$ & $0.88 \mathrm{e}-04$ \\
$\sigma$ & $3.52 \mathrm{e}-04$ & $0.46 \mathrm{e}-04$ \\
$e_{2}$ & $0.74 \mathrm{e}-03$ & $14.19 \mathrm{e}-03$ \\
$D_{\mathrm{KL}}(\mathcal{P} \| \mathcal{Q})$ & $1.16 \mathrm{e}-02$ & $15.18 \mathrm{e}-02$ \\
\hline
\end{tabular}

Table 4: Root mean-square error, mean, standard deviation, and Kullback-Leibler divergence from a Gaussian distribution of the lift-induced and spurious drag coefficients computed by $\ell_{1}-$ minimization with $N=102$ sampling points.

\section{Conclusions}

In this paper we have applied a non intrusive technique to reconstruct surrogates (response surfaces) for the uncertainty quantification and robust 


\begin{tabular}{|c||c|c|}
\hline & $C_{D, \text { lift induced }}$ & $C_{D, \text { spurious }}$ \\
\hline \hline $\mathscr{S}_{1}$ & $0.004 \mathrm{e}-01$ & $9.417 \mathrm{e}-01$ \\
$\mathscr{S}_{2}$ & $4.811 \mathrm{e}-01$ & $0.005 \mathrm{e}-01$ \\
$\mathscr{S}_{3}$ & $0.014 \mathrm{e}-01$ & $0.001 \mathrm{e}-01$ \\
$\mathscr{S}_{4}$ & $0.007 \mathrm{e}-01$ & $0.021 \mathrm{e}-01$ \\
$\mathscr{S}_{5}$ & $0.000 \mathrm{e}-01$ & $0.002 \mathrm{e}-01$ \\
$\mathscr{S}_{6}$ & $0.082 \mathrm{e}-01$ & $0.056 \mathrm{e}-01$ \\
$\mathscr{S}_{7}$ & $0.071 \mathrm{e}-01$ & $0.013 \mathrm{e}-01$ \\
$\mathscr{S}_{8}$ & $0.020 \mathrm{e}-01$ & $0.012 \mathrm{e}-01$ \\
$\mathscr{S}_{9}$ & $0.096 \mathrm{e}-01$ & $0.010 \mathrm{e}-01$ \\
$\mathscr{S}_{10}$ & $0.044 \mathrm{e}-01$ & $0.008 \mathrm{e}-01$ \\
\hline
\end{tabular}

Table 5: Main-effect sensitivity indices of the output quantities of interest computed by $\ell_{1}-$ minimization with $N=102$ sampling points.
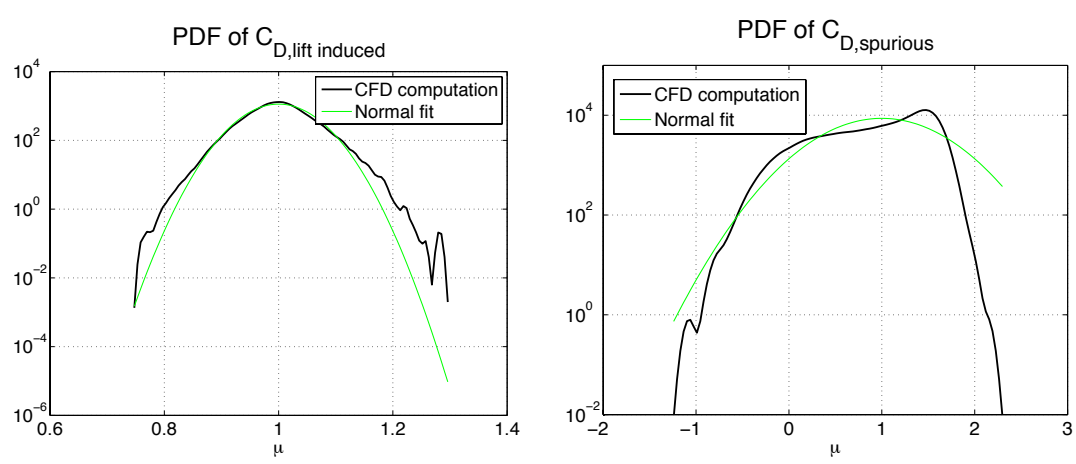

Figure 16: PDFs of the lift-induced (left) and spurious (right) drag coefficients computed by $\ell_{1}$-minimization with $N=102$ sampling points. Comparison with a standard Gaussian distribution (green curve) with the same mean and standard deviation.

optimization of aeroelastic computations, taking the example of the ALBATROS configuration. This technique is a regression approach, whereby the 
surrogates are polynomial chaos expansions in the variable parameters space. It typically uses structured sampling set, e.g. Gauss quadratures, to compute the expansion coefficients in this basis. However we have shown in this study that invoking a "sparsity-of-effects" feature, the framework of compressed sensing is applicable to the present case. Accordingly, an $\ell_{1}-$ minimization algorithm has been implemented to reconstruct polynomial surrogates in a regression approach. In addition, it requires unstructured sampling sets orders of magnitude smaller than the ones required by the widespread sampling rules (Shannon's sampling theorem or Gauss quadratures, for example), and typically smaller than the dimension of the polynomial space where the surrogates are sought for. The procedure is also non-adaptive in the sense that it identifies both the amplitude of the leading expansion coefficients and their order in the polynomial basis. At last, its application to the aeroelastic database of the present study has clearly demonstrated its ability to handle a parameters space of high dimension-thus alleviating to some extent the "curse of dimensionality." Therefore, the sparse reconstruction technique outlined here constitutes a promising direction for future developments in large-scale industrial applications involving complex geometries and flows.

\section{Acknowledgment}

This research has received funding from the European Union's Seventh Framework Programme for research, technological development and demonstration under grant agreement no. ACP3-GA-2013- 60503 (UMRIDA Project WwW. umrida.eu). 


\section{References}

[1] R. Arnaud, F. Poirion. Optimization of an uncertain aeroleastic system using stochastic gradient approaches. AIAA J. Aircraft, 51(3):1061-1066 (2014).

[2] K. J. Badcock, S. Timme, S. Marques, H. Khodaparast, M. Prandina, J. E. Mottershead, A. Swift, A. Da Ronch, M. A. Woodgate. Transonic aeroelastic simulation for instability searches and uncertainty analysis. Prog. Aerosp. Sci., 47(5):392-423 (2011).

[3] D. Bailly. LHS optimized sampling. Personnal communication (2015).

[4] P. Beran, B. Stanford, C. Schrock. Uncertainty quantification in aeroelasticity. Annu. Rev. Fluid Mech., 49:361-386 (2017).

[5] G. Blatman, B. Sudret. An adaptive algorithm to build up sparse polynomial chaos expansions for stochastic finite element analysis. Prob. Engng. Mech., 25(2):183-197 (2010).

[6] R. Cameron, W. Martin. The orthogonal development of nonlinear functionals in series of Fourier-Hermite functionals. Ann. Math., 48(2):385$392(1947)$.

[7] L. Cambier, S. Heib, S. Plot. The ONERA elsA CFD software: input from research and feedback from industry. Mechanics 8 Industry, 14(3):159-174 (2013). 
[8] E. J. Candès, J. K. Romberg, T. Tao. Stable signal recovery from incomplete and inaccurate measurements. Commun. Pure Appl. Math., 59(8):1207-1223 (2006).

[9] E. Candès, J. K. Romberg. Sparsity and incoherence in compressive sampling. Inverse Probl., 23(3):969-985 (2007).

[10] E. J. Candès, M. B. Wakin. An introduction to compressive sampling. IEEE Sig. Proc. Mag., 25(2):21-30 (2008).

[11] G. Carrier, O. Atinault, S. Dequand, J.-L. Hantrais-Gervois, C. Liauzun, B. Paluch, A.-M. Rodde, C. Toussaint. Investigation of a strut-braced wing configuration for future commercial transport. 28th Congress of the International Council of the Aeronautical Sciences (ICAS 2012), 23-28 September 2012, Brisbane. Paper ICAS 2012-1.10.2 (2012).

[12] S. C. Castravete, R. A. Ibrahim. Effect of stiffness uncertainties on the flutter of a cantilever wing. AIAA J., 46(4): 925-935 (2008).

[13] S. C. Chen, D. L. Donoho, M. A. Saunders. Atomic decomposition by basis pursuit. SIAM J. Sci. Comput., 20(1):33-61 (1998).

[14] D. Clouteau, É. Savin, D. Aubry. Stochastic simulations in dynamic soil-structure interaction. Meccanica, 36(4):379-399 (2001).

[15] Y. Dai, C. Yang. Methods and advances in the study of aeroelasticity with uncertainties. Chin. J. Aeronaut., 27(3):461-474 (2014).

[16] B. J. Debusschere, H. N. Najm, P. P. Pébay, O. M. Knio, R. G. Ghanem, O. P. Le Maître. Numerical challenges in the use of polynomial chaos rep- 
resentations for stochastic processes. SIAM J. Sci. Comput., 26(2):698$719(2005)$.

[17] D. Destarac. Far-field/near-field drag balance and applications of drag extraction in CFD. CFD-Based Aircraft Drag Prediction and Reduction, Lecture Series 2003-02, Von Karman Institute for Fluid Dynamics (2003).

[18] D. Destarac, J. van der Vooren. Drag/thrust analysis of jet-propelled transonic transport aircraft; Definition of physical drag components. Aerosp. Sci. Technol., 8(6):545-556 (2004).

[19] D. L. Donoho. Compressed sensing. IEEE Trans. Inform. Theory, 52(4):1289-1306 (2006).

[20] A. Doostan, H. Owhadi. A non-adapted sparse approximation of PDEs with stochastic inputs. J. Comput. Phys., 230(8):3015-3034 (2011).

[21] http://elsa.onera.fr. Online the March 20th, 2019.

[22] O. G. Ernst, A. Mugler, H.-J. Starkloff, E. Ullman. On the convergence of generalized polynomial chaos expansions. Math. Model. Num. Anal., 46(2):317-339 (2012).

[23] T. Gerstner, M. Griebel. Numerical integration using sparse grids. $\mathrm{Nu}$ mer. Algorithms, 18(3-4):209-232 (1998).

[24] R. Ghanem. Ingredients for a general purpose stochastic finite elements implementation. Comput. Methods Appl. Mech. Engng., 168(1-4):19-34 (1999). 
[25] R. G. Ghanem, P. D. Spanos. Stochastic Finite Elements: A Spectral Approach. Springer, New York NY (1991).

[26] I. Ghazlane, G. Carrier, A. Dumont, J.-A. Désidéri. Aerostructural adjoint method for flexible wing optimization. 53rd AIAA/ASME/ASCE/AHS/ASC Structures, Structural Dynamics, and Materials Conference, 23-26 April 2012, Honolulu HI. AIAA Paper \#2012-1924 (2012).

[27] I. Ghazlane. Adjoint-based aerostructural sensitivity analysis for wing design. PhD Thesis, Université Nice-Sophia Antipolis, December 2012.

[28] D. M. Ghiocel, R. G. Ghanem. Stochastic finite-element analysis of seismic soil-structure interaction. ASCE J. Engng. Mech., 128(1):66-77 (2002).

[29] J. Hampton, A. Doostan. Compressive sampling of polynomial chaos expansions: Convergence analysis and sampling strategies. J. Comput. Phys., 280:363-386 (2015).

[30] J.-L. Hantrais-Gervois, D. Destarac. Drag polar invariance with flexibility. AIAA J. Aircraft, 52(3):997-1001 (2015).

[31] S. Hosder, R. W. Walters, M. Balch. Efficient uncertainty quantification applied to the aeroelastic analysis of a transonic wing. 46th AIAA Aerospace Sciences Meeting and Exhibit, 7-10 January 2008, Reno NV. AIAA Paper \#2008-0729 (2008).

[32] A. Jameson. Transonic flow calculations. MAE-report 1651, Princeton University (1983). 
[33] E. T. Jaynes. Information theory and statistical mechanics. Phys. Rev., 106(4):620-630 (1957).

[34] E. T. Jaynes. Information theory and statistical mechanics II. Phys. Rev., 108(2):171-190 (1957).

[35] S. Kullback, R. A. Leibler. On information and sufficiency. Ann. Math. Statist., 22(1):79-86 (1951).

[36] J. Kuttenkeuler, U. Ringertz. Aeroelastic tailoring considering uncertainties in material properties. Struct. Optimization, 15(3-4):157-162 (1998).

[37] O. Le Maître, O. Knio. Spectral Methods for Uncertainty Quantification. With Applications to Computational Fluid Dynamics. Springer, Dordrecht (2010).

[38] J. Le Meitour, D. Lucor, J.-C. Chassaing. Prediction of stochastic limit cycle oscillations using an adaptive Polynomial Chaos method. J. Aeroel. Struct. Dyn., 2(1):3-22 (2012).

[39] M. Marcelet. Implementation of shape optimization method coupling computational fluid dynamics and computational structural mechanics. PhD Thesis, ENSAM ParisTech, December 2008.

[40] L. Mathelin, K. A. Gallivan. A compressed sensing approach for partial differential equations with random input data. Commun. Comput. Phys., 12(4):919-954 (2012). 
[41] M. Méheut, A. Arntz, G. Carrier. Aerodynamic shape optimizations of a blended wing body configuration for several wing planforms. 30th AIAA Applied Aerodynamics Conference, 25-28 June 2012, New Orleans LA. AIAA Paper \#2012-3122 (2012).

[42] Q. Mo, S. Li. New bounds on the restricted isometry constant $\delta_{2 k}$. Appl. Comput. Harm. Anal., 31(3):460-468 (2011).

[43] H. N. Najm. Uncertainty quantification and polynomial chaos techniques in computational fluid dynamics. Annu. Rev. Fluid Mech., 41:35-52 (2009).

[44] E. Novak, K. Ritter. Simple cubature formulas with high polynomial exactness. Constructive Approx., 15(4):499-522 (1999).

[45] J. Peng, J. Hampton, A. Doostan. A weighted $\ell_{1}-$ minimization approach for sparse polynomial chaos expansions. J. Comput. Phys., 267:92-111 (2014).

[46] C. L. Pettit. Uncertainty quantification in aeroelasticity: Recent results and research challenges. AIAA J. Aircraft, 41(5):1217-1229 (2004).

[47] F. Poirion. Effect of structural uncertainties on flutter analysis. Rech. Aérosp., 1992-6:31-41 (1992).

[48] H. Rauhut, R. Ward. Sparse Legendre expansions via $\ell_{1}$-minimization. J. Approx. Th., 164(5):517-533 (2012).

[49] É. Savin, A. Resmini, J. Peter. Sparse polynomial surrogates for aerodynamic computations with random inputs. 18th AIAA Non-Deterministic 
Approaches Conference, 4-8 January 2016, San Diego CA. AIAA Paper \#2016-0433 (2016).

[50] É. Savin, B. Faverjon. Computation of higher-order moments of generalized polynomial chaos expansions. Int. J. Num. Methods Engng., 111(12):1192-1200 (2017).

[51] D. Schiavazzi, A. Doostan, G. Iaccarino. Sparse multiresolution regression for uncertainty quantification. Int. J. Uncertainty Quantification, 4(4):303-331 (2014).

[52] S. A. Smolyak. Quadrature and interpolation formulas for tensor products of certain classes of functions. Soviet Math. Dokl., 4:240-243 (1963).

[53] C. Soize, R. G. Ghanem. Physical systems with random uncertainties: chaos representations with arbitrary probability measure. SIAM J. Sci. Comput., 26(2):395-410 (2004).

[54] P. R. Spalart, S. R. Allmaras. A one-equation turbulence model for aerodynamic flows. 30th AIAA Aerospace Sciences Meeting and Exhibit, 6-9 January 1992, Reno NV. AIAA Paper \#1992-0439 (1992).

[55] B. Sudret. Global sensitivity analysis using polynomial chaos expansions. Reliab. Eng. Syst. Safe., 93(7):964-979 (2008).

[56] T.-C. Sun. A finite element method for random differential equations with random coefficients. SIAM J. Numer. Anal., 16(6):1019-1035 $\underline{(1979) .}$ 
[57] E. van den Berg, M. P. Friedlander. SPGL1: A solver for large-scale sparse reconstruction. June 2007.

[58] E. van den Berg, M. P. Friedlander. Probing the Pareto frontier for basis pursuit solutions. SIAM J. Sci. Comput., 31(2):890-912 (2008).

[59] M. P. Wand, M. D. Jones. Kernel Smoothing. Chapman and Hall/CRC, Boca Raton FL (1995).

[60] G. Wang. Adaptive response surface method using inherited Latin hypercube design points. ASME J. Mech. Des., 125(2):210-220 (2003).

[61] N. Wiener. The homogeneous chaos. Amer. J. Math., 60(4):897-936 $\underline{(1938) .}$

[62] J. A. S. Witteveen, S. Sarkar, H. Bijl. Modeling physical uncertainties in dynamic stall induced fluid-structure interaction of turbine blades using arbitrary polynomial chaos. Comput. Struct., 85(11-14):866-878 (2007).

[63] D. Xiu, G. E. Karniadakis. The Wiener-Askey polynomial chaos for stochastic differential equations. SIAM J. Sci. Comput., 24(2):619-644 (2002).

[64] D. Xiu, J. S. Hesthaven. High-order collocation methods for differential equations with random inputs. SIAM J. Sci. Comput., 27(3):1118-1139 $\underline{(2005) .}$

[65] S.-K. Yoon, A. Jameson. An LU-SSOR scheme for the Euler and NavierStokes equations. 25th AIAA Aerospace Sciences Meeting, 12-15 January 1987, Reno NV. AIAA Paper \#1987-0600 (1987). 